\title{
Bifurcation from a normally degenerate manifold
}

\author{
D.R.J. Chillingworth and L. Sbano
}

\begin{abstract}
Local bifurcation theory typically deals with the response of a degenerate but isolated equilibrium state or periodic orbit of a dynamical system to perturbations controlled by one or more independent parameters, and characteristically uses tools from singularity theory. There are many situations, however, in which the equilibrium state or periodic orbit is not isolated but belongs to a manifold $S$ of such states, typically as a result of continuous symmetries in the problem. In this case the bifurcation analysis requires a combination of local and global methods, and is most tractable in the case of normal nondegeneracy, that is when the degeneracy is only along $S$ itself and the system is nondegenerate in directions normal to $S$.

In this paper we consider the consequences of relaxing normal nondegeneracy, which can generically occur within 1-parameter families of such systems. We pay particular attention to the simplest but important case where $\operatorname{dim} S=1$ and where the normal degeneracy occurs with corank 1 . Our main focus is on uniform degeneracy along $S$, although we also consider aspects of the branching structure for solutions when the degeneracy varies at different places on $S$. The tools are those of singularity theory adapted to global topology of $S$, which allow us to explain the bifurcation geometry in natural way. In particular, we extend and give a clear geometric setting for earlier analytical results of Hale and Taboas.
\end{abstract}

\section{The general problem}

\subsection{Introduction}

Let $F_{0}: M \rightarrow T M$ be a smooth ${ }^{\dagger}$ vector field on a smooth (possibly infinite-dimensional) manifold $M$, with the property that the flow generated by $F_{0}$ has a smooth $d$-dimensional manifold $S$ of equilibria with finite $d>0$. In other words, $F_{0}$ vanishes everywhere on $S$. We now perturb $F_{0}$ to

$$
F_{\varepsilon}:=F_{0}+\varepsilon \mathcal{F}+O\left(|\varepsilon|^{2}\right)
$$

where $\mathcal{F}$ is a smooth vector field on a neighbourhood of $S$ in $M$ and where $\varepsilon$ is a real parameter, and we ask what can be said about zeros of $F_{\varepsilon}$ close to $S$ in $M$ for $\varepsilon$ sufficiently small but nonzero. In particular, we ask which points $x_{0} \in S$ are such that a branch of equilibria emanates from them as $\varepsilon$ varies away from zero.

More generally we may consider a multiparameter deformation $F_{\varepsilon}$ where $\varepsilon \in \mathbb{R}^{q}$, as in $[\mathbf{3 1}][\mathbf{4 2}]$ or $[\mathbf{1 1}, \mathbf{1 3}]$ for example. In this case the term $\varepsilon \mathcal{F}$ in $(1.1)$ is interpreted as $\sum_{i=1}^{q} \varepsilon_{i} \mathcal{F}_{i}$ for a collection $\left\{\mathcal{F}_{1}, \ldots, \mathcal{F}_{q}\right\}$ of smooth vector fields, and $O\left(|\varepsilon|^{2}\right)$ means terms of degree 2 or higher

2000 Mathematics Subject Classification 34C23, 34C30, 58K05, 58K35, 58K40.

Acknowledgements. The authors are grateful for partial support from the London Mathematical Society through a Scheme 4 grant, ref. 4433. We are indebted to David Kirby for helpful conversations about the algebra in Section 4.2 .

$\dagger$ By smooth we mean $C^{\infty}$. However, it is clear that many of our results hold with only a few orders of differentiability. 
in the components of $\varepsilon$. Here it is useful to think in terms of equilibrium branches as $\varepsilon$ moves away from the origin in a given direction in parameter space $\mathbb{R}^{q}$, and we discuss this more carefully below. Of course, it is not clear a priori that branching behaviour depends only on $\mathcal{F}$ and not on the $O\left(|\varepsilon|^{2}\right)$ terms, although it is a key aspect of our results that often under suitable generic ${ }^{\ddagger}$ assumptions on $\mathcal{F}$ this is indeed the case.

In many situations where bifurcation from a manifold arises in applications the manifold $S$ is normally non-degenerate with respect to $F_{0}$, meaning that at each point $x \in S$ the tangent space $T_{x} S$ (which automatically lies in $\operatorname{ker} T_{x} F_{0}$ ) in fact coincides with $\operatorname{ker} T_{x} F_{0}$, so that $T_{x} F_{0}$ is as nondegenerate as possible under the given geometric assumptions. Bifurcation under these conditions has been well studied: see $[\mathbf{2}],[\mathbf{1 3}],[\mathbf{1 6}],[\mathbf{1 9}],[\mathbf{3 1}],[\mathbf{4 2}]$. A standard technique is to show by a global Lyapunov-Schmidt reduction along $S$ that for small $\varepsilon$ there is a smooth manifold $S_{\varepsilon}$ close to $S$ (viewed as the image of a small section of the normal bundle $N S$ of $S$ in $M$ ) such that the 'normal' component of $F_{\varepsilon}$ vanishes on $S_{\varepsilon}$. This reduces the problem to a study of the 'tangential' component of $F_{\varepsilon}$, which corresponds to a tangent vector field $\tilde{F}_{\varepsilon}$ on $S$ via the projection $N S \rightarrow S$. The sought-for zeros of $F_{\varepsilon}$ close to $S$ then correspond to the zeros of the vector field $\tilde{F}_{\varepsilon}$ on $S$ itself. $^{\dagger}$

In the absence of further information the number of zeros of $\tilde{F}_{\varepsilon}$ can often be given a lower bound by topological means such as index theory: see the techniques of $[\mathbf{1 9}, \mathbf{2 0}]$ for example. In the variational case where $F_{\varepsilon}$ is the gradient field of a real-valued function the same may be assumed for $\tilde{F}_{\varepsilon}$ (see Section 2.1) and the usual tools are Lyusternik-Schnirelmann category theory in general or Morse theory if critical points are assumed to be nondegenerate: see for example $[\mathbf{2}],[\mathbf{9}],[\mathbf{3 9}],[\mathbf{2 0}],[\mathbf{1 8}]$ and $[43],[3,4]$ for applications to Hamiltonian systems. With further information on symmetries $[\mathbf{2 1}],[\mathbf{3 6}],[\mathbf{7}],[\mathbf{4 2}]$ or on the actual perturbing field $\mathcal{F}$ more specific results can be obtained as in $[\mathbf{1 0}$, $11,12,13]$ and $[14,15,16]$.

The purpose of this paper is to investigate the consequences of relaxing the normal non-degeneracy condition by supposing that $T_{x} S$ is a proper subspace of $\operatorname{ker} T_{x} F_{0}$, that is

$$
\operatorname{dim} \operatorname{ker} T_{x} F_{0}=k+d
$$

for all $x \in S$, where $k \geq 1$ does not depend on $x$. We call this normal degeneracy with constant corank $k$. Our most detailed results are for the simplest (but important) case when $k=1$, but some of the methods and observations are applicable also to the general case.

1.1.1. The group orbit case. There is a natural situation in which manifolds of equilibria arise automatically, namely where the manifold $S$ is the orbit of the action of a Lie group $\Gamma$ under which $F_{0}$ is equivariant. In particular this occurs when $F_{0}$ is the gradient of a $\Gamma$-invariant function $f_{0}: M \rightarrow \mathbb{R}$. Problems with $\Gamma$-symmetry arise naturally in classical mechanics [1] although $\Gamma$-orbits of relative equilibria (equilibria modulo the group action) play a more significant role there than do manifolds of equilibria themselves. The tools for handling bifurcations of equilibria in this setting, such as the local decomposition results of Krupa [33] or the extensive theory of branching patterns due to Field $[\mathbf{2 5}, \mathbf{2 6}]$, deal with spontaneous symmetry-breaking where the symmetry of the problem as a whole is preserved (although individual solutions may have less symmetry). This machinery

\footnotetext{
$\ddagger$ In our context a generic condition or assumption is one which holds for an open dense set of the maps or objects under consideration. Making this precise requires formal machinery of transversality in jet spaces and techicalities which we mostly prefer to avoid: in our cases the interpretation of genericity is straightforward.

${ }^{\dagger}$ For a more careful description see Section 2.
} 
is not applicable to the problems that we consider, however, as the perturbed system $F_{\varepsilon}$ does not share the symmetry ( $\Gamma$-equivariance) of $F_{0}$; our problems go under the general heading of forced symmetry-breaking.

In problems formulated on function spaces of periodic functions, there is a natural circular symmetry defined by the phase-shift, and in mechanical systems natural symmetries arise from coordinate invariance. Translational symmetry can also play a key role in bifurcation theory of differential equations on the real line: see [34] for example.

For a typical group orbit we expect normal non-degeneracy, but if $F_{0}$ is a member of a smooth 1parameter family of vector fields $F_{0}^{\gamma}$ each of which is $\Gamma$-equivariant then, since (for square matrices) dropping rank by 1 is a codimension- 1 phenomenon, we would expect to find isolated values of the parameter $\gamma \in \mathbb{R}$ for which the corresponding equilibrium manifold $S^{\gamma}$ is normally degenerate with constant corank 1 . The constancy of corank in this case is a consequence of the equivariance.

1.1.2. Non-constant corank. In contrast with the above, non-degeneracy may fail in that $\operatorname{dim} \operatorname{ker} T_{x} F_{0}>\operatorname{dim} S$ only at isolated points $x \in S$. This does not arise in the group orbit case, since the group action implies that the behaviour at all points of $S$ is 'the same'. Analysis of this type of degeneracy requires different techniques, which we touch upon in Section 5. See also [38].

1.1.3. Sources of the problem. The break-up of families of periodic orbits of Hamiltonian systems is a classical problem, first treated in a modern spirit in the fundamental papers of Weinstein [43] and Moser [36]. Abstract treatment of bifurcation from a manifold was pioneered by Hale [31], with applications to planar forced ordinary differential equations, and extended by Hale and Taboas [32] to the case of normal degeneracy. Further important techniques and results relating particularly to symmetries were set out by Dancer $[\mathbf{1 9}, \mathbf{2 0}, \mathbf{2 1}]$ and Vanderbauwhede [42], again supposing normal nondegeneracy. We mention also the results of Fiedler et al. [27] on the dynamics of a vector field in a neighbourhood of a line of equilibria: there, though, the equilibria are required to persist throughout. Applications of bifurcation from a manifold were exploited by Ambrosetti et al. $[\mathbf{3}, \mathbf{4}]$ in the context of Hamiltonian systems and also more generally [2].

Meanwhile, a general geometric framework for multiparameter bifurcation from a normally nondegenerate manifold was formulated by the first author $[\mathbf{1 0}, \mathbf{1 1}, \mathbf{1 3}]$, and customised tools adapted to families of periodic orbits of ordinary differential equations were systematically refined in a series of papers by Chicone $[\mathbf{1 4}, \mathbf{1 5}, \mathbf{1 6}]$. It is the work of Hale et al. and Chicone that is the main inspiration for the current paper.

1.1.4. Relevance of the examples. We illustrate our general technique through two main examples. The first one comes from Hamiltonian systems and is motivated by the search for periodic orbits in a fixed energy level. This problem is widely studied and has a vast literature. An outline of various possible approaches can be found in Sbano [41]. Our method is in the spirit of $[\mathbf{3}, \mathbf{4}]$, although for us the novel feature is the normal degeneracy of the initial rotationally symmetric periodic orbit.

Our second example originates from a typical class of equations employed to study chemical reaction networks. Usually these systems are formed by polynomial and rational vector fields, in the presence of degeneracies due to conservation relations. For this reason in the literature special techniques have been developed (see Domijan [23] for a recent review). We show that our method gives a general framework for studying these systems and can be successfully employed in perturbation problems. 


\subsection{Outline of the approach}

An overview of our approach in this paper is as follows. First we apply Lyapunov-Schmidt reduction to enable us to focus on the core of the problem. As a consequence of normal degeneracy, the reduced problem still involves a $k$-dimensional normal component of the vector field, as well as the automatic $d$-dimensional tangential component. We would like to 'unfold' this degenerate $(k+d)$-dimensional vector field, using $\varepsilon$ as a multi-parameter, but we are unable to use standard tools of unfolding (deformation) theory directly as the degeneracy along $S$ causes the local problem to have infinite codimension. Therefore we make appropriate generic assumptions about the behaviour of the deformation term $\mathcal{F}$ along the manifold $S$. With this, we can in principle describe generic geometry of such deformations, at least for small $k$. In the fundamental case $k=1$ we are able to give a rather complete picture.

In Section 2 we formulate the initial reduction process, and in Section 3 we show how to adapt the standard notion of versal deformation to our context, giving particular attention to the simplest nontrivial low-dimensional case. In Section 4 we combine global geometry with local algebra to describe generic branching structure, and in Section 5 we study local branching structure in closer detail. Finally, in Section 6 we discuss three areas of application of the methods set out in this paper.

\section{Lyapunov-Schmidt reduction}

Let $N S$ be a normal bundle for $S$ in $M$, that is a vector sub-bundle of $T_{S} M:=\left.T M\right|_{S}$ orthogonal to $T S$ (using a Riemannian structure $\nu$ on $M$ ). A neighbourhood of the zero section of $N S$ can be taken to parametrise a tubular neighbourhood of $S$ in $M$ via the exponential map associated to $\nu$, and so then we may without loss of generality regard the whole bifurcation problem as taking place on a neighbourhood $W$ of the zero section of the bundle $N S$.

Let $H$ be a connection on $N S$, that is a (smooth) sub-bundle of $T N S$ orthogonal to (vectors tangent to) the fibres of $N S$ : thus

$$
T_{w} N S=H_{w} \oplus T_{w} N_{x}=H_{w} \oplus N_{x}
$$

for all $w$ lying in the fibre $N_{x}$ of $N S$ over $x \in S$. This enables us to define a linear bundle map $\pi: T N S \rightarrow N S$ as projection onto the second factor in each tangent subspace $T_{w} N S$.

Given a vector field $F: W \subset N S \rightarrow T N S$ the composition $\pi F: W \rightarrow N S$ satisfies $\pi F(W \cap$ $\left.N_{x}\right) \subset N_{x}$ for each $x \in S$. Thus $\pi F$ is a 'vertical' vector field on $W$ obtained by projecting $F$ parallel to the 'horizontal' subspaces determined by $H$. If $w \in W \cap N_{x}$ we write $D F(w) \in \operatorname{Lin}\left(N_{x}, N_{x}\right)$ to denote the 'vertical' derivative of $F$ (i.e. the derivative of $F \mid N_{x}$ ) at $w$.

Let $F_{\varepsilon}: M \rightarrow T M, \varepsilon \in \mathbb{R}^{q}$ be a vector field as in Section 1 , with $F_{0}(x)=0$ for all $x \in S$. As above, we replace $M$ by $W \subset N S$. Since $T_{x} S \subset \operatorname{ker} T_{x} F_{0}$ for all $x \in S$, the constant corank hypothesis (1.2) implies that at the origin $0_{x}$ of $N_{x}$ the kernel $K_{x}:=\operatorname{ker} D F_{0}\left(0_{x}\right)$ is a $k$-dimensional linear subspace of $N_{x}$ for every $x \in S$. We now make the following assumptions:

(A1) The subspaces $\left\{K_{x}\right\}$ together define a smooth $k$-dimensional vector sub-bundle $K$ of $N S$.

(A2) There is a smooth codimension- $k$ vector sub-bundle $L=\left\{L_{x}\right\}$ of $N S$ complementary to $K$ in $N S$; thus $N_{x}=K_{x} \oplus L_{x}$ for all $x \in S$. 
(A3) There is a smooth vector bundle decomposition $N S=P \oplus R$ such that $R_{x}$ is the range of $D F_{0}\left(0_{x}\right)$ and $\operatorname{dim} P_{x}=k$ for all $x \in S$.

Remark. In finite dimensions the smoothness of $K$ holds automatically, given the constant dimension $k$, since the kernel of a matrix is the orthogonal complement of the range of its transpose: thus if $D F_{0}\left(0_{x}\right)$ varies smoothly with $x$ then a basis for $\mathbb{R}^{n}$ orthogonal to the rows of $D F_{0}\left(0_{x}\right)$ can also be chosen to vary smoothly. The construction of $L$ and $P$ is straightforward.

Smoothness of $K$ also holds in many naturally-arising infinite-dimensional cases where $D F_{0}\left(0_{x}\right)$ is a differential operator, so that its kernel consists of the solutions $\xi(t)$ to a $k$-dimensional system of linear differential equations; construction of $L$ and $P$ is also often easy in such cases.

If $D F_{0}\left(0_{x}\right)$ is self-adjoint (as for a gradient vector field, for example) then we may take $L_{x}=R_{x}$ and $P_{x}=K_{x}$.

Let $S_{0}$ denote the (image of the) zero section of the bundle $K$. Of course $S_{0}=S$, but the $S_{0}$ notation will focus attention on $S$ as a submanifold of $K$. Applying Lyapunov-Schmidt reduction globally along a neighbourhood of $S_{0}$ in $K$ we obtain the following result.

Proposition 2.1. There exists a neighbourhood $U$ of $S_{0}$ in $K$ with the property that for all sufficiently small $\varepsilon$ there is a unique smooth bundle map $\sigma^{\varepsilon}: U \rightarrow L$ such that

(i) $\sigma^{0} \mid S_{0}=0$ and

(ii) the $R$-component of $F_{\varepsilon}$ vanishes precisely at the points lying on the graph $U^{\varepsilon} \subset N S$ of $\sigma^{\varepsilon}$.

Proof. Let $r$ denote the bundle projection $N S=P \oplus R \rightarrow R$, and consider the composition $r \circ$ $\pi F_{\varepsilon}: W \rightarrow R$. When $\varepsilon=0$ the vertical derivative of this smooth map at $0_{x} \in N_{x}$ (identified with $\left.x \in S_{0}\right)$ is by definition of $R$ a linear surjection with finite-dimensional kernel $K_{x}$. Hence by the Implicit Function Theorem (IFT), given $x_{0} \in S_{0}$ there is a neighbourhood $V$ of $x_{0}$ in $K$ and $\varepsilon_{0}>0$ such that for all $|\varepsilon|<\varepsilon_{0}$ and $x \in V \cap S_{0}$ the solution set to $r \circ \pi F_{\varepsilon}=0$ in the fibre $N_{x}$ is the intersection with $N_{x}$ of the graph $V^{\varepsilon}$ of a unique smooth bundle map $\sigma_{V}^{\varepsilon}: V \rightarrow L$. See Figure 1. The construction of $\sigma^{\varepsilon}$ on a global neighbourhood $U$ of $S_{0}$ from local sections $\sigma_{V}^{\varepsilon}$ then follows by uniqueness of $\sigma_{V}^{\varepsilon}$ on $V$ and the local compactness of $S$.

Since the $(d+k)$-dimensional manifold $U^{\varepsilon}$ is precisely where the vertical component of the vector field $F_{\varepsilon}$ has zero $R$-component, it follows that the zeros of $F_{\varepsilon}$ are the zeros of the vector field on $U^{\varepsilon}$ obtained by projecting $F_{\varepsilon}$ to $T S \oplus P$. (Note that this projected vector field is not necessarily tangent to $U^{\varepsilon}$.) The problem therefore distils to that of solving the reduced problem

$$
\tilde{F}_{\varepsilon}(u):=(\mathbf{1}-r \circ \pi) F_{\varepsilon}\left(u, \sigma^{\varepsilon}(u)\right)=0 \in T S \oplus P, \quad u \in U \subset K
$$

where 1 denotes the identity map on $T M=T N S$. Here if $w=\left(u, \sigma^{\varepsilon}(u)\right) \in N_{x}$ we identify $H_{w}$ with $T_{x} S$ through the natural bundle projection $T N S \rightarrow T S$, so that $\mathbf{1}-r \circ \pi$ is projection on $T S \oplus P$.

Remark. If $F$ is of class $C^{p}$ then $\tilde{F}$ is also $C^{p}$ for $1 \leq p \leq \infty, \omega$.

\subsection{The gradient case}

In the important special case when $F_{\varepsilon}$ is the gradient $\nabla f_{\varepsilon}$ of a real-valued function $f_{\varepsilon}$ (with respect to the Riemannian structure $\nu$ ) then the zeros of $\tilde{F}_{\varepsilon}$ correspond to the zeros of a gradient 


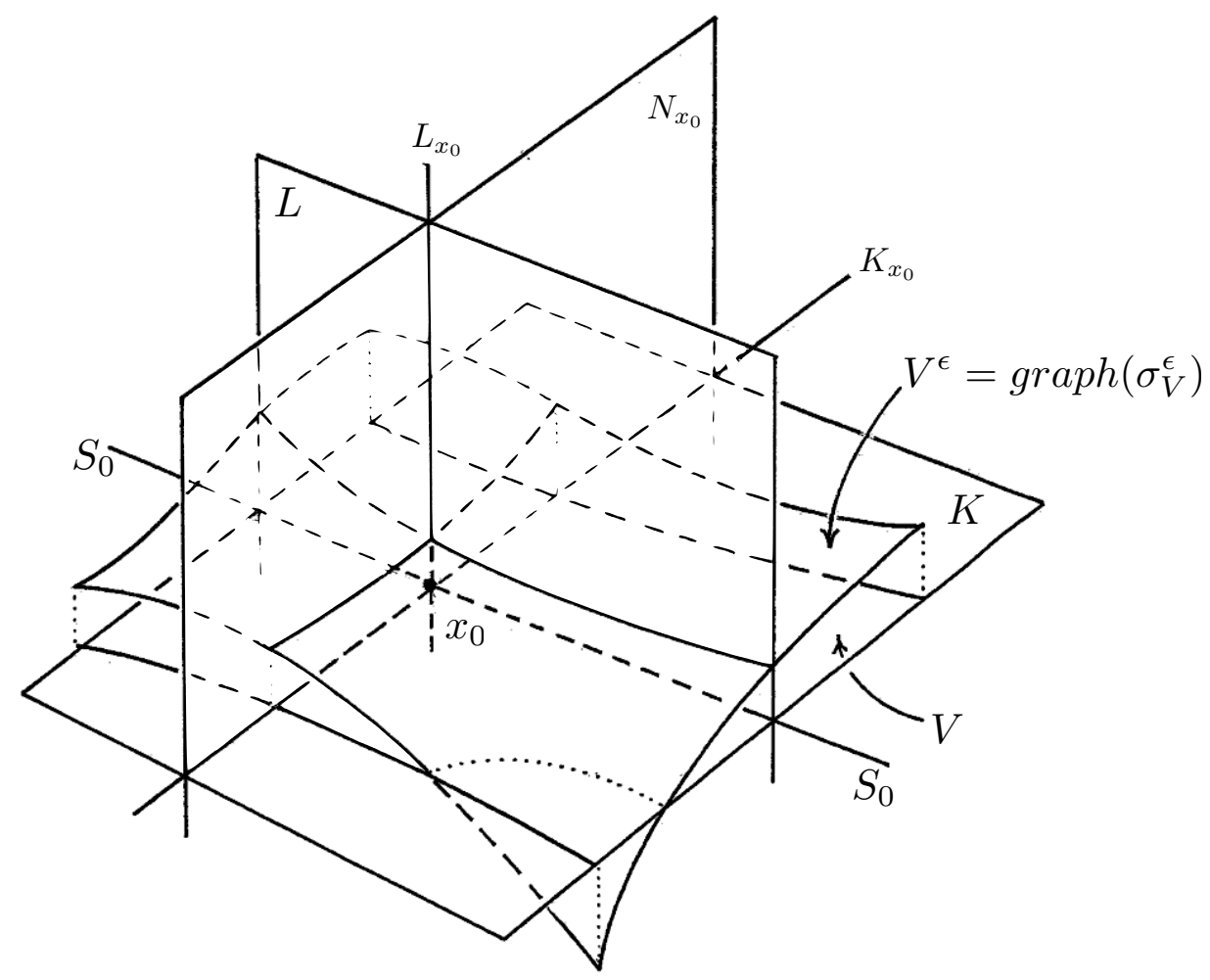

Figure 1. The geometry of Lyapunov-Schmidt reduction at $x_{0} \in S_{0}$. Here $N$ is the normal bundle to $S=S_{0}$ in $M$ and $K_{x_{0}}=\operatorname{ker} D F\left(x_{0}\right)$.

field on $U$. To see this, take $R=L$ and $P=K$ (see earlier remark) and observe that if $u \in U$ and $\tilde{f}_{\varepsilon}(u):=f_{\varepsilon}\left(u, \sigma^{\varepsilon}(u)\right)$ then

$$
\begin{aligned}
\nabla \tilde{f}_{\varepsilon}(u) & =(\mathbf{1}-r \circ \pi) \nabla f_{\varepsilon}\left(u, \sigma^{\varepsilon}(u)\right) \\
& =\tilde{F}_{\varepsilon}(u),
\end{aligned}
$$

there being no contribution to $\nabla \tilde{f}_{\varepsilon}(u)$ from the second argument of $f_{\varepsilon}$ because $r \circ \pi \nabla f_{\varepsilon}$ vanishes identically on $U^{\varepsilon}$ by construction.

\subsection{Interpretation of the reduced problem}

The reduced vector field $\tilde{F}_{\varepsilon}: U \subset K \rightarrow T S \oplus P$ satisfies

$$
\tilde{F}_{0}\left(0_{x}\right)=0 \quad \text { for all } x \in S
$$

and

$$
D \tilde{F}_{0}\left(0_{x}\right)=0 \quad \text { for all } x \in S,
$$

since $F_{0}=0$ and $\sigma^{0}=0$ on $S_{0}$, and also $(1-r \circ \pi) D F_{0}\left(0_{x}\right)=0$ by definition of $r$. We can therefore regard $\tilde{F}_{\varepsilon}$ as arising from a family of smooth singular map germs

$$
\tilde{F}_{0} \mid K_{x}: K_{x}, 0_{x} \rightarrow T_{x} S \oplus P_{x}
$$


parametrised (globally) by $x \in S$ which is then perturbed (locally) by $\varepsilon \in \mathbb{R}^{q}$ for $|\varepsilon|$ small. After shrinking the original neighbourhood $U$ if necessary, we may write

$$
\tilde{F}_{\varepsilon}(u)=\tilde{F}_{0}(u)+\varepsilon \widetilde{\mathcal{F}}(\varepsilon, u)
$$

with $u \in U \subset K$ and $\varepsilon \in \mathbb{R}^{q}$ by analogy with the original problem in Section 1 . Note that $\tilde{F}_{\varepsilon}\left(0_{x}\right)$ is not necessarily zero when $\varepsilon \neq 0$.

\subsection{Uniform degeneracy}

It may happen that the map germ $\tilde{F}_{0} \mid K_{x}$ is in some precise sense independent of $x \in S$ : we shall make this more explicit in Section 3 below. In this case we say the normal degeneracy is uniform. For example, in the group orbit case with trivial isotropy (no nontrivial element of $\Gamma$ keeps $x \in S$ fixed) the action of $\Gamma$ provides a natural coordinate system for the whole of $N S$ as a product bundle over $S$. In these coordinates the decompositions $N_{x}=K_{x} \oplus L_{x}=P_{x} \oplus R_{x}$ are independent of $x \in S$ and uniformity in a natural sense follows automatically.

Machinery to study perturbations of functions and maps is provided by singularity theory. In the next section we introduce the key ideas that will be required for our purposes.

\section{Versal deformations}

For ease of exposition we make a simplifying assumption, namely that all of the bundles over $S$ that are involved in the construction of the reduced vector field $\tilde{F}_{\varepsilon}$ (including the tangent bundle $T S$ are trivial bundles. This is automatically the case if $S$ is contractible (a copy of $\mathbb{R}$ for example) and is usually the case in applications when $S$ is a circle. Even when the assumption fails, we can apply the methods below to contractible open subsets of $S$ and piece together the results subsequently. We remark later on how the methods would need to be adapted to apply intrinsically to nontrivial bundles.

Let us therefore take $K=S \times \mathbb{R}^{k}$ and $T S \oplus P=S \times \mathbb{R}^{d} \times \mathbb{R}^{k}$ so that as in Section 2.2 we regard $\tilde{F}_{\varepsilon}$ as a family of smooth map germs

$$
\tilde{F}_{\varepsilon}(x, \cdot): \mathbb{R}^{k}, 0 \rightarrow \mathbb{R}^{d+k}
$$

parametrised by $x \in S$ and (small) $\varepsilon \in \mathbb{R}^{q}$. Since we are here interested in the zero locus

$$
Z_{\tilde{F}}=\left\{(\varepsilon, x, y) \in \mathbb{R}^{q} \times S \times \mathbb{R}^{k}: \tilde{F}_{\varepsilon}(x, y)=0 \in \mathbb{R}^{d+k}\right\}
$$

for small $|y|$ rather than other geometric features of $\tilde{F}_{\varepsilon}$ as a map, we are free to apply coordinate changes in a neighbourhood of $0 \times S_{0}=0 \times S \times 0$ that respect the geometry of the zero locus and the role of $\varepsilon$ as a parameter. A natural class of coordinate transformations to use is the class of contact equivalences ${ }^{\dagger}$ or $\mathcal{K}$-equivalences. Singularity theory provides powerful tools for handling classification and geometry of map germs up to $\mathcal{K}$-equivalence, and the essence of this paper is the adaptation of those techniques to our class of bifurcation problem. It is in order to use the tools of singularity theory that we assume $C^{\infty}$ smoothness of all data in the problem. Background material for the singularity theory in this section can be found in a range of texts such as $[\mathbf{5}, \mathbf{6}, \mathbf{2 8}]$ and

\footnotetext{
$\dagger$ Not to be confused with contact transformations from classical mechanics.
} 
from a slightly different viewpoint in $[\mathbf{3 0}]$.

\subsection{Basic versal deformation}

We here introduce the main elements of the theory of $\mathcal{K}$-versal deformations of map germs that will be used in our analysis. To recall the concepts we shall use generic symbols $x$ and $F$ that are not the same as $x \in S$ and the map $F_{\varepsilon}$ of our main problem.

Definition 3.1. Two smooth map germs $f, g: \mathbb{R}^{n}, 0 \rightarrow \mathbb{R}^{p}, 0$ are $\mathcal{K}$-equivalent if there is a diffeomorphism germ $\phi$ at the origin in $\mathbb{R}^{n}$ and an invertible $p \times p$ matrix $A$ depending smoothly on $x \in \mathbb{R}^{n}$ such that ${ }^{\dagger}$

$$
g(x) \equiv A(x) f(\phi(x))
$$

Clearly the zero set for $f$ is the image under $\phi$ of the zero set for $g$. Since $A$ is invertible any degeneracy of $f$ at the origin is 'the same' as that of $g$.

Definition 3.2. An $r$-parameter deformation of a map germ $f: \mathbb{R}^{n}, 0 \rightarrow \mathbb{R}^{p}, 0$ is map germ $F: \mathbb{R}^{r} \times \mathbb{R}^{n},(0,0) \rightarrow \mathbb{R}^{p}, 0$ such that $F(0, x) \equiv f(x)$.

To keep notation simple we shall write $F$ as a map, the fact that it represents a map germ at $(0,0)$ being understood.

Now for the key definition through which we classify types of bifurcation behaviour.

Definition 3.3. Two deformations $F_{1}, F_{2}: \mathbb{R}^{r} \times \mathbb{R}^{n} \rightarrow \mathbb{R}^{p}$ of $f$ are $\mathcal{K}$-equivalent if there is a diffeomorphism germ $\Phi$ at the origin in $\mathbb{R}^{r} \times \mathbb{R}^{n}$ of the form

$$
\Phi(a, x)=(\psi(a), \phi(a, x))
$$

and an invertible $p \times p$ matrix $A$ varying smoothly with respect to $(a, x) \in \mathbb{R}^{r} \times \mathbb{R}^{n}$ such that

$$
F_{2}(a, x) \equiv A(a, x) F_{1} \circ \Phi(a, x) .
$$

Note that $F_{1}, F_{2}$ are $\mathcal{K}$-equivalent as maps, but the coordinate change $\Phi$ distinguishes between the roles of the variables $x$ and parameters $a$. In particular, $\Phi$ takes the zero locus $F_{2}^{-1}(0)$ to $F_{1}^{-1}(0)$, and $\psi$ takes the parameter-space projection of $F_{2}^{-1}(0)$ to that of $F_{1}^{-1}(0)$.

In the case when $A(x)$ or $A(a, x)$ is the identity matrix (hence can be deleted from the expressions (3.1) or (3.3)) the maps or deformations are right equivalent ( $\mathcal{R}$-equivalent). It is easy to check that if two real-valued functions are $\mathcal{R}$-equivalent (up to a constant) then their gradient vector fields are $\mathcal{K}$-equivalent.

\footnotetext{
†The symbol $\equiv$ indicates the two map germs are identical: it is not an equation for $x$.
} 
Much of the power of singularity theory resides in the central notion of versal deformation: a typical map germ has an identifiable class of deformations from which all others can be obtained by suitable coordinate substitution.

Definition 3.4. The $r$-parameter deformation $F$ of $f$ is $\mathcal{K}$-versal if, given any $q$-parameter deformation $G(c, x)$ of $f$, there is a map germ $\alpha: \mathbb{R}^{q}, 0 \rightarrow \mathbb{R}^{r}, 0$ with the property that $G$ is $\mathcal{K}$ equivalent to the deformation

$$
\alpha^{*} F:(c, x) \mapsto F(\alpha(c), x) .
$$

The deformation $F$ is $\mathcal{K}$-miniversal if there exist no $\mathcal{K}$-versal deformations of $f$ with fewer parameters.

In other words, the parameters $a_{i}$ in the $\mathcal{K}$-versal deformation $F$ can be expressed as suitable (smooth) functions $\alpha_{i}$ of the given parameters $c_{j}$ in order to recover $G$ from $F$ up to $\mathcal{K}$-equivalence. Note that in general $\alpha$ is not a diffeomorphism, since (apart from other considerations) $q$ and $r$ need not be equal.

Remark. It is a consequence of the general theory that if $F$ is $\mathcal{K}$-miniversal then $F(a, \cdot)$ and $f$ are $\mathcal{K}$-equivalent germs if and only if $a=0 \in \mathbb{R}^{r}$.

If $f$ is nonsingular (that is $D f(0)$ has maximal rank) then the IFT implies that every deformation of $f$ is $\mathcal{K}$-versal. In what follows we therefore suppose that $f$ is singular.

Not every $f$ necessarily has a $\mathcal{K}$-versal deformation, but the failure to do so is (in a precise sense) exceptional. Moreover, and most importantly, $\mathcal{K}$-versal deformations when they exist can be chosen to take the explicit form

$$
F(a, x) \equiv a_{1} v_{1}(x)+\ldots+a_{r} v_{r}(x)+f(x)
$$

for a certain (nonunique) collection of polynomial map germs $v_{i}: \mathbb{R}^{n} \rightarrow \mathbb{R}^{p}, 1 \leq i \leq r$, that can be constructed from $f$ by a standard algebraic procedure.

From Definitions 3.2 and 3.4 we deduce the following:

Proposition 3.1. The zero set $Z_{F}:=F^{-1}(0)$ is a 'standard object' in $\mathbb{R}^{r} \times \mathbb{R}^{n}$ associated with $f$ : the zero locus $Z_{G}$ of any given deformation $G$ of $f$ as above is (up to diffeomorphism $\Phi$ ) the inverse image of $Z_{F}$ under the map $(c, x) \mapsto(\alpha(c), x)$.

We are concerned with bifurcations, so the points of $Z_{G}$ of particular interest to us are those where the projection of $Z_{G}$ into parameter space $\mathbb{R}^{q}$ fails to be a submersion: these are points where the local structure of the set of solutions $x$ to $G(c, x)=0$ can change as $c$ varies locally.

Definition 3.5. The singular set $\Sigma_{G}$ of the deformation $G: \mathbb{R}^{q} \times \mathbb{R}^{n} \rightarrow \mathbb{R}^{p}$ is defined as

$$
\Sigma_{G}=\left\{(c, x) \in \mathbb{R}^{q} \times \mathbb{R}^{n}: \operatorname{rank} D_{x} G(c, x)<p\right\} .
$$

The discriminant $\Delta_{G}$ is the projection of $Z_{G} \cap \Sigma_{G}$ into the parameter space $\mathbb{R}^{q}$. 
Thus $\Delta_{G}$ is the set of $c \in \mathbb{R}^{q}$ for which there exists some $x \in \mathbb{R}^{n}$ with $G(c, x)=0$ but $D_{x} G(c, x)$ has rank less than $p$.

The importance of $\Sigma_{G}$ for us lies in the following key result much used in deformation (unfolding) theory. See e.g. $[\mathbf{2 8}$, p.49] or $[\mathbf{1 7}$, Ch.11,(18)] for a proof.

Proposition 3.2. If $G$ is a submersion, so $Z_{G}$ is a smooth submanifold of $\mathbb{R}^{q} \times \mathbb{R}^{n}$ of codimension $p$, then $\Sigma_{G}$ is the set of singular points of the projection $Z_{G} \subset \mathbb{R}^{q} \times \mathbb{R}^{n} \rightarrow \mathbb{R}^{q}$.

Remark. If $n<p$ (a case important to us) then $\Sigma_{G}=Z_{G}$ and the rank condition is vacuous.

From Definition 3.4 it follows that if $F$ is a $\mathcal{K}$-miniversal deformation of $f$ then, for any other deformation $G$ of $f$, the discriminant $\Delta_{G}$ is (up to diffeomorphism $\psi$ ) the inverse image of the discriminant $\Delta_{F}$ under the parameter map $\alpha$. The discriminant $\Delta_{F}$ is also a 'standard object' and for polynomial $f$ it is given by a finite set of polynomial equations in $\mathbb{R}^{r}$.

The policy statement below is the ideological basis for our geometrical treatment of bifurcation from $S$ in this paper:

The key to understanding the geometry of the zero locus of the deformation $G$ of $f$ is to understand the geometry of the associated map $\alpha$ from the given parameter space of $G$ into that of a $\mathcal{K}$-miniversal deformation $F$ of $f$, and in particular the intersection of the image of $\alpha$ with the discriminant $\Delta_{F}$. Generic assumptions about this intersection lead to conclusions about generic geometry of $\Delta_{G}$.

Because of the degeneracy along $S$ we shall need to take extra care in implementing this policy.

\subsection{Deformation along a manifold.}

There is a vital technical issue to deal with, before we can exploit the above ideas. In our context as described in Section 2.2 the full parameter space is $\mathbb{R}^{q} \times S$, with the $\varepsilon$-deformation terms vanishing on $0 \times S$. However, the notion of versal deformation as described above applies only in a neighbourhood of a single point in parameter space, whereas we would like to apply it in a consistent way over a whole neighbourhood of $0 \times S$ in $\mathbb{R}^{q} \times S$.

In standard proofs $[\mathbf{6}, \mathbf{2 8}]$ of the existence of $\mathcal{K}$-versal deformations the requisite coordinate changes are constructed by integrating suitably chosen vector fields, the existence of these vector fields being established by using the Preparation Theorem for smooth function germs. However, as pointed out in [6] (see Section 4.5, Example 4), there is a parametrised version of the Preparation Theorem which holds where the parameter belongs to a closed interval $[0,1]$ and where (as in our case of uniform normal degeneracy) the map germ to be unfolded is independent of the parameter. The proof of this theorem uses (as expected) a smooth partition of unity. The same proof applies equally well to a parameter belonging to any smooth compact manifold (there are no topological obstructions in view of the linear nature of the constructions). This enables us to work with the 'global' parameter $(\varepsilon, x) \in \mathbb{R}^{q} \times S$ for $|\varepsilon|$ small. We can therefore state the following deformation result, customised for our purposes:

Proposition 3.3. Let $h: \mathbb{R}^{k}, 0 \rightarrow \mathbb{R}^{d+k}, 0$ be a smooth map germ which has a $\mathcal{K}$-versal deformation $H: \mathbb{R}^{r} \times \mathbb{R}^{k} \rightarrow \mathbb{R}^{d+k}$. Let $W$ be a neighbourhood of $0 \times S_{0}$ in $\mathbb{R}^{q} \times S \times \mathbb{R}^{k}$ and let $F: W \rightarrow \mathbb{R}^{d+k}$ be a smooth map such that $F(0, x, y)=h(y)$ for all $x \in S$. Then there exists a 
neighbourhood $W^{\prime} \subset W$ of $0 \times S_{0}$ and a smooth map

$$
\Phi: W^{\prime} \rightarrow \mathbb{R}^{r} \times \mathbb{R}^{k}:(\varepsilon, x, y) \mapsto(\psi(\varepsilon, x), \phi(\varepsilon, x, y)),
$$

as well as a nonsingular matrix $A(\varepsilon, x, y)$ of size $d+k$ and defined on $W^{\prime}$, such that

(i) the identity

$$
F(\varepsilon, x, y) \equiv A(\varepsilon, x, y) H \circ \Phi(\varepsilon, x, y)
$$

holds for all $(\varepsilon, x, y) \in W^{\prime}$

(ii) the map $\mathbb{R}^{k} \rightarrow \mathbb{R}^{k}: y \mapsto \tilde{y}=\phi(\varepsilon, x, y)$ is a diffeomorphism for each $(\varepsilon, x) \in \mathbb{R}^{q} \times S$, with $\phi(0, x, y)=y$ for all $x \in S$

(iii) $\psi(0, x)=0 \in \mathbb{R}^{r}$ for all $x \in S$.

Putting together Proposition 3.3 and the expression (3.4) we now obtain the deformation result we require for application to the reduced bifurcation problem $\tilde{F}_{\varepsilon}=0$ as in (2.1).

COROLlary 3.4. Let the vector field $F_{0}$ satisfy uniform normal degeneracy on $S$ as in Section 2.3, so that in coordinates $(x, y)$ on the neighbourhood $U$ of $S_{0}$ in $K=S \times \mathbb{R}^{k}$ we have $\tilde{F}_{0}(x, y) \cong h(y)$ where $\tilde{F}_{\varepsilon}$ is the reduced map as in (2.1). If $h$ has $\mathcal{K}$-versal deformation of the form

$$
H(a, y) \equiv a_{1} v_{1}(y)+\ldots a_{r} v_{r}(y)+h(y)
$$

then, up to premultiplication by a nonsingular matrix depending smoothly on $(\varepsilon, x, y)$, we can express $\tilde{F}_{\varepsilon}$ in the form

$$
\tilde{F}_{\varepsilon}(x, y)=\tilde{F}(\varepsilon, x, y) \equiv a_{1}(\varepsilon, x) v_{1}(\tilde{y})+\ldots+a_{r}(\varepsilon, x) v_{r}(\tilde{y})+h(\tilde{y}) \in \mathbb{R}^{d+k}
$$

where $y \mapsto \tilde{y}$ is a diffeomorphism germ at the origin in $\mathbb{R}^{k}$ depending (smoothly) on $(\varepsilon, x) \in \mathbb{R}^{q} \times S$ and where also

$$
a_{i}(0, x) \equiv 0
$$

for all $x \in S$ and $i=1,2, \ldots, r$.

\subsection{Global and local geometry of the discriminant.}

We continue to assume uniform degeneracy, and without loss of generality we take $\tilde{F}_{\varepsilon}$ to be of the form (3.7) where after the coordinate change $y \rightarrow \tilde{y}$ we drop the tilde on $y$. The identity (3.8) implies that we can write

$$
a_{i}(\varepsilon, x)=b_{i}(x) \varepsilon+c_{i}(x)(\varepsilon, \varepsilon)+O\left(|\varepsilon|^{3}\right) \in \mathbb{R}
$$

for $i=1,2, \ldots, r$, where $b_{i}(x), c_{i}(x)$ are linear and bilinear maps $\mathbb{R}^{q} \rightarrow \mathbb{R}$ and $\mathbb{R}^{q} \times \mathbb{R}^{q} \rightarrow \mathbb{R}$ respectively. Thus

$$
b_{i}(x) \varepsilon=\sum_{j} b_{i j}(x) \varepsilon_{j} \quad \text { and } \quad c_{i}(x)(\varepsilon, \varepsilon)=\sum_{l m} c_{i l m}(x) \varepsilon_{l} \varepsilon_{m}
$$

where

$$
b_{i j}(x)=\left.\frac{\partial a_{i}}{\partial \varepsilon_{j}}(\varepsilon, x)\right|_{\varepsilon=0} \quad \text { and } \quad c_{i l m}(x)=\left.\frac{\partial^{2} a_{i}}{\partial \varepsilon_{l} \partial \varepsilon_{m}}(\varepsilon, x)\right|_{\varepsilon=0}
$$


We next write $\varepsilon$ in 'polar coordinates' as

$$
\varepsilon=\rho s, \quad s \in \mathbb{S}^{q-1}, \quad \rho \in \mathbb{R}_{+}
$$

where $\mathbb{R}_{+}=\{t \in \mathbb{R}: t \geq 0\}$ and $\mathbb{S}^{q-1}=\left\{s \in \mathbb{R}^{q}:|s|=1\right\}$. From (3.9) the smooth map

$$
a: \mathbb{R}^{q} \times S \rightarrow \mathbb{R}^{r}:(\varepsilon, x) \mapsto\left(a_{1}(\varepsilon, x), \ldots, a_{r}(\varepsilon, x)\right)
$$

satisfies

$$
a_{i}(\varepsilon, x)=\rho b_{i}(x) s+\rho^{2} c_{i}(x)(s, s)+O\left(\rho^{3}\right) \in \mathbb{R}
$$

for $i=1, \ldots, r$. We study the geometry of the map

$$
b: \mathbb{S}^{q-1} \times S \rightarrow \mathbb{R}^{r}:(s, x) \mapsto\left(b_{1}(x) s, \ldots, b_{r}(x) s\right) .
$$

From this we obtain the geometry of the map $a: \mathbb{R}^{q} \times S \rightarrow \mathbb{R}^{r}$ to first order in $\rho$ as the cone on that of $b$. We anticipate that under suitable generic assumptions on $b$ this cone structure will enable us to capture the true geometry of the discriminant

$$
\Delta_{\tilde{F}}:=a^{-1}\left(\Delta_{H}\right) \in \mathbb{R}^{q} \times S
$$

of $\tilde{F}$ near the origin.

To investigate this closely we look at rays of the cone and their intersections with $\Delta_{H}$ as $\rho \rightarrow 0$. It will be convenient to do this using a 'blow-up' or (in reverse) pinching technique as follows. First define the pinching map

$$
\tau: \mathbb{R} \times \mathbb{S}^{q-1} \rightarrow \mathbb{R}^{q}:(\rho, s) \mapsto(\rho s)
$$

and write

$$
\tilde{\tau}:=\tau \times i d: \mathbb{R} \times \mathbb{S}^{q-1} \times S \rightarrow \mathbb{R}^{q} \times S,
$$

noting that since $\tau(\rho,-s)=\tau(-\rho, s)$ we may restrict attention to $\rho \in \mathbb{R}_{+}:=\{r \in \mathbb{R}: r \geq 0\}$ if convenient. Next, let

$$
D_{\tilde{F}}^{1}:=b^{-1}\left(\Delta_{H}\right) \subset \mathbb{S}^{q-1} \times S .
$$

Generic assumptions on $b$ will enable us to characterise the local structure of $D_{\widetilde{F}}^{1}$ which then determines local models for the structure of

$$
\Delta_{\tilde{F}}^{1}:=\alpha^{-1}\left(\Delta_{H}\right) \subset \mathbb{R}_{+} \times \mathbb{S}^{q-1} \times S
$$

where

$$
\alpha:=a \circ \tilde{\tau}: \mathbb{R}_{+} \times \mathbb{S}^{q-1} \times S \rightarrow \mathbb{R}^{r} .
$$

(Note that here $\alpha$ plays the role of the map $\alpha$ in Definition 3.4.) Finally, the application of $\tilde{\tau}$ gives the local geometry of $\Delta_{\tilde{F}}=a^{-1}\left(\Delta_{H}\right)$.

The following elementary fact about $\tau$ will be important.

Proposition 3.5. Let $\gamma: \mathbb{R}, 0 \rightarrow \mathbb{R} \times \mathbb{S}^{q-1},\left(0, x_{0}\right)$ be (the germ of) a smooth path such that

$$
\gamma(t)=\left(p t^{m}+O\left(t^{m+1}\right), s_{0}+t u+O\left(t^{2}\right)\right)
$$

where $p \neq 0 \in \mathbb{R}$ and $u \neq 0 \in T_{s_{0}} \mathbb{S}^{q-1}$ (so $u \perp s_{0}$ in $\mathbb{R}^{q}$ ). Then the image of $\delta:=\tau \circ \gamma$ in $\mathbb{R}^{q}$ is (close to the origin) the union of two $C^{1}$ arcs from the origin, each having contact of order $(m+1) / m$ with their common tangent direction $s_{0}$. 
Proof. Immediate, since $\tau \gamma(t)=p t^{m} s_{0}+O\left(t^{m+1}\right) s_{0}+p t^{m+1} u+O\left(t^{m+2}\right)$, so taking the parametrisation $t^{\prime}=t^{m}$ gives

$$
\tau \gamma\left(t^{\prime}\right)-p s_{0} t^{\prime}=p t^{\prime(m+1) / m} u
$$

to lowest order in $t^{\prime}$.

Observe that if $m$ is even then $\delta$ has a cusp at the origin in $\mathbb{R}^{q}$, while if $m$ is odd then $\delta$ is a $C^{1}$ 1-manifold passing through the origin.

The following geometrical construction will also play a key part in our investigations.

Let $H: \mathbb{R}^{r} \times \mathbb{R}^{n} \rightarrow \mathbb{R}^{p}$ be a $\mathcal{K}$-miniversal deformation of the singular germ $h: \mathbb{R}^{n}, 0 \rightarrow \mathbb{R}^{p}, 0$.

Definition 3.6. A vector $v \in \mathbb{R}^{r}$ is called a positive tangent vector to $\Delta_{H}$ at the origin if there exists $t_{0}>0$ and a differentiable path $\gamma:\left[0, t_{0}\right) \rightarrow \mathbb{R}^{r}$ with $\gamma(0)=0 \in \mathbb{R}^{r}$ such that

(i) $\gamma(t) \in \Delta_{H}$ for all $t \in\left[0, t_{0}\right)$

(ii) $\gamma^{\prime}(0)=v$.

The positive tangent cone $T_{H}$ for $\Delta_{H}$ at the origin is the union of all the positive tangent vectors at the origin.

We now turn to applying this machinery to the reduced bifurcation problem (2.1).

\subsection{Branch points and bifurcation}

As $\varepsilon \in \mathbb{R}^{q}$ moves away from the origin in a given direction we expect branches of solutions to $\tilde{F}_{\varepsilon}=0$ to emanate from certain points $(x, 0) \in S_{0}$.

Definition 3.7. A solution branch for $\tilde{F}$ at $x \in S$ is a differentiable path $z:\left[0, t_{0}\right) \rightarrow Z_{\tilde{F}} \subset$ $\mathbb{R}^{q} \times S \times \mathbb{R}^{k}$ for some $t_{0}>0$ such that

(i) $z^{\prime}(0) \neq 0$

(ii) $z(0)=(0, x, 0) \in \mathbb{R}^{q} \times S_{0}$

(iii) $z(t) \in \mathbb{R}^{q} \times S_{0}$ only if $t=0$.

A solution branch $z$ has direction $s \in \mathbb{S}^{q-1}$ if the projection of $z^{\prime}(0)$ into $\mathbb{R}^{q}$ is nonzero and in the direction $s$; otherwise we say the branch $z$ is tangent to $S$. If a solution branch exists at $x \in S$ with direction $s$ then we say that $x$ is a branch point with branch direction $s$.

Recall (see the Remark after Definition 3.5) that since $k<d+k$ the zero set $Z_{\tilde{F}}$ is here the same as the singular set $\Sigma_{\tilde{F}}$ and projects to $\Delta_{\tilde{F}}=a^{-1}\left(\Delta_{H}\right) \subset \mathbb{R}^{q}$.

From the geometry of $\Delta_{H}$ we obtain a necessary condition for branching.

Proposition 3.6. A necessary condition for $x_{0} \in S$ to be a branch point with branch direction $s_{0} \in \mathbb{S}^{q-1}$ is that $b\left(s_{0}, x_{0}\right)$ lie in the positive tangent cone $T_{H}$ for the discriminant $\Delta_{H}$ of the $\mathcal{K}$-versal deformation $H$ of $h$. 
Proof. Let $z$ be a solution branch at $x_{0} \in S$ with direction $s_{0}$ so that

$$
z(t)=(\tilde{z}(t), y(t))=(\rho(t) s(t), x(t), y(t)) \in \mathbb{R}^{q} \times S \times \mathbb{R}^{k}
$$

where $\rho(0)=0, \rho^{\prime}(0) \neq 0$ and $s(0)=s_{0}, x(0)=x_{0}$. From (3.10) we have

$$
\left.\frac{d}{d t} a(\tilde{z}(t))\right|_{t=0}=\frac{d}{d t} \rho(t) b(s(t), x(t))+\left.O\left(\rho(t)^{2}\right)\right|_{t=0}=\rho^{\prime}(0) b\left(s_{0}, x_{0}\right)
$$

and so $b\left(s_{0}, x_{0}\right) \in T_{H}$ since $a(\tilde{z}(t)) \in \Delta_{H}$ for $t \in\left[0, t_{0}\right)$.

In the examples considered below the set $T_{H}$ turns out to be either a hyperplane or a halfhyperplane. Generically we expect the map $b: \mathbb{S}^{q-1} \times S \rightarrow \mathbb{R}^{r}$ to be transverse to $T_{H}$ in an appropriate sense, and the transversality allows us to establish the existence of branch points and to describe the local solution structure regardless of the terms $O\left(\varepsilon^{2}\right)$ in $a(\varepsilon, x)$.

Because of the technical complications of the relevant singularity theory, the approach described above is useful in practice only for small values of $d$ and/or $k$. However, the case $k=1$ is most significant, as it represents the least departure from normal nondegeneracy of $S$, while the case $d=1$ naturally occurs in the study of periodic orbits of differential equations where there is an automatic circle group action on the space of periodic functions (see Section 6). Therefore the simplest but most important case $d=k=1$ merits detailed investigation.

\section{The fundamental case of dimension 1 and corank 1 .}

Here we have a 1-dimensional equilibrium manifold $S$ of constant corank $k=1$. Throughout this section we continue to assume that the normal degeneracy is uniform; some branching analysis applying to the general case is given below in Section 5 .

To simplify notation we drop the tilde throughout and write (3.7) as

$$
F_{\varepsilon}(x, y)=F(\varepsilon, x, y) \equiv g(\varepsilon, x, y)+h(y) \in \mathbb{R}^{2}
$$

with multiparameter $\varepsilon \in \mathbb{R}^{q}$ and $u=(x, y) \in S \times \mathbb{R}$ since here $k=1$ and $d+k=2$. Each component of $h(y)$ is without constant or linear terms because of the normal degeneracy of the zero set $S_{0}$. Supposing that at least one component has a nonzero Taylor series at 0 , it is a straightforward exercise (see $[\mathbf{2 8}]$ for example) to check that $h$ is $\mathcal{K}$-equivalent to the germ $y \mapsto\left(0, y^{m}\right)$ for some integer $m>0$. We first take the least degenerate case $m=2$ and study this example in detail in the geometric framework outlined above. We then show how this approach extends to general $m$ and illustrate in particular how it recovers and sets in a geometric context the analysis of this problem for $q=2$ given by Hale and Taboas $[\mathbf{3 2}]$.

\subsection{The case $m=2:$ quadratic degeneracy.}

Here

$$
F(\varepsilon, x, y)=g(\varepsilon, x, y)+h(y)=g(\varepsilon, x, y)+\left(0, y^{2}\right) \in \mathbb{R}^{2}
$$

for $(x, y) \in S \times \mathbb{R}$ with $\operatorname{dim} S=1$, and $\varepsilon \in \mathbb{R}^{q}$.

4.1.1. Geometry of the zero set. A $\mathcal{K}$-miniversal deformation of $h(y) \equiv\left(0, y^{2}\right)$ is

$$
H(a, y)=H\left(a_{1}, a_{2}, a_{3}, y\right)=\left(a_{1}+a_{2} y, a_{3}+y^{2}\right)
$$


(see $[\mathbf{6}],[\mathbf{2 8}]$ ) and so by Corollary 3.4 up to $\mathcal{K}$-equivalence we can suppose $F$ to have the form

$$
F(\varepsilon, x, y)=\left(a_{1}(\varepsilon, x)+a_{2}(\varepsilon, x) y, a_{3}(\varepsilon, x)+y^{2}\right)
$$

for $|y|,|\varepsilon|$ sufficiently small and for all $x \in S$, where $a(0, x)=0 \in \mathbb{R}^{3}$ for all $x \in S$. As in Section 3.3, in order to describe the bifurcation behaviour of $F$ we need to understand the geometry of the discriminant $\Delta_{F}=a^{-1}\left(\Delta_{H}\right) \subset \mathbb{R}^{q} \times S$ where (as is easily seen by eliminating $y$ from $H(a, y)=0$ ) the discriminant $\Delta_{H}$ is given explicitly by

$$
a_{1}^{2}+a_{2}^{2} a_{3}=0
$$

This is the equation of a Whitney umbrella in $\mathbb{R}^{3}$ : see Figure 2.

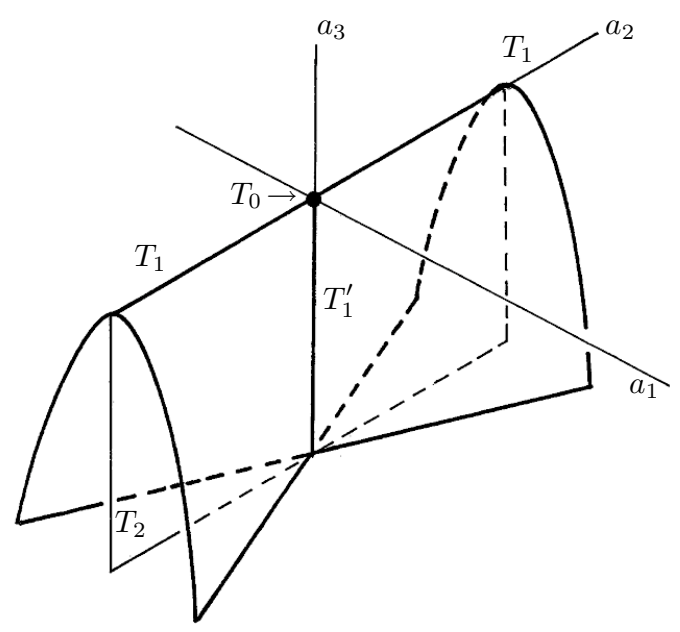

Figure 2. Whitney umbrella: locus $a_{1}^{2}+a_{2}^{2} a_{3}=0$ showing the stratification $\left\{T_{0}, T_{1}, T_{1}^{\prime}, T_{2}\right\}$.

From Section 3.3, the key intermediate step is to study the geometry of

$$
D_{F}^{1}:=b^{-1}\left(\Delta_{H}\right) \in \mathbb{S}^{q-1} \times S
$$

where $b: \mathbb{S}^{q-1} \times S \rightarrow \mathbb{R}^{3}$ is as in (3.11). Following Proposition 3.6 we first identify the set $b^{-1}(T)$ where $T=T_{H}$ is the positive tangent cone to $\Delta_{H}$ at the origin. In the present case

$$
T=\left\{a \in \mathbb{R}^{3}: a_{1}=0, a_{3} \leq 0\right\}
$$

since any smooth path in $\Delta_{H}$ from the origin can be checked to have contact of order at least $\frac{3}{2}$ with the plane $a_{1}=0$.

We now make the following generic hypothesis, which is at the heart of our whole approach to bifurcating from a normally degenerate manifold:

(H1) The map $b: \mathbb{S}^{q-1} \times S \rightarrow \mathbb{R}^{3}$ is transverse to $T$. 
The notion of transversality to a variety such as $T$ rather than to a smooth manifold assumes a stratification of the variety, that is a suitable decomposition of the variety into finitely many manifolds of varying dimension, the smaller comprising the boundary of the larger. See $[\mathbf{5}, \mathrm{Ch} .3, \S 1]$ for example. In this case there is an obvious and natural stratification $\left\{T_{0}, T_{1}, T_{2}\right\}$ where $T_{0}$ is the origin, $T_{1}$ is the $a_{2}$-axis with $a_{2} \neq 0$, and $T_{2}$ is the part of the $\left(a_{2}, a_{3}\right)$-plane with $a_{3}<0$ : here $\operatorname{dim} T_{i}=i$ for $i=0,1,2$. However, we see from Figure 2 that since our ultimate interest is in geometry of $\Delta_{F}$ we should also take account of the fact that the negative $a_{3}$-axis $T_{1}^{\prime}$ is a natural 1-dimensional stratum in $\Delta_{H}$. We hence include this half-line $T_{1}^{\prime}$ as a 1-dimensional stratum for $T$ when interpreting the hypothesis (H1).



Figure 3. Schematic representation of $b^{-1}(T)$ (dashed curve) and $D_{F}^{1}=b^{-1}\left(\Delta_{H}\right)$ (solid curve) in the case $d=k=1, n=q=2$ (Whitney umbrella) showing end points (black dots) and intersection points (white dots). The whole of $D_{F}^{1}$ lies in a strip of width $\pi$ between two parallel curves and has quadratic tangency with those at the end points. The 1-dimensional manifolds $S$ and $\mathbb{S}^{1}$ are each represented as closed intervals.

Since $\Delta_{H}$ and $T$ are 2-dimensional objects in $\mathbb{R}^{3}$ the essential geometry of transversality is captured by taking $q=1$ or 2 so that $\operatorname{dim}\left(\mathbb{S}^{q-1} \times S\right)=1$ or 2 . We first study carefully the case $q=2$, and comment on the cases $q=1$ and $q \geq 3$ later.

4.1.2. The 'typical' case $q=2$. We take $S$ compact for ease of exposition; adjustments to the noncompact case are straightforward.

The transversality hypothesis (H1) implies that $b^{-1}(T)$ consists of a smooth 1-manifold with boundary, thus a finite collection of closed arcs or embedded circles in $\mathbb{S}^{1} \times S$. The set of end 
points of these arcs is $b^{-1}\left(T_{1}\right)$, while interior points constitute $b^{-1}\left(T_{2}\right)$ with possible intersection points $b^{-1}\left(T_{1}^{\prime}\right)$. Note from the geometry of $\Delta_{H}$ and linearity of $b(s, x)$ in $s$ that if $(s, x)$ is an end point then so is $(-s, x)$. Moreover, the intersection of $S^{1} \times\{x\}$ with $\Delta_{F}^{1}$ lies in the semicircular arc $\left\{s \in S^{1}: b_{3}(s, x) \leq 0\right\}$ since all of $\Delta_{H}$ lies in the half-space $a_{3} \leq 0$. A schematic representation of this geometry is shown in Figure 3.

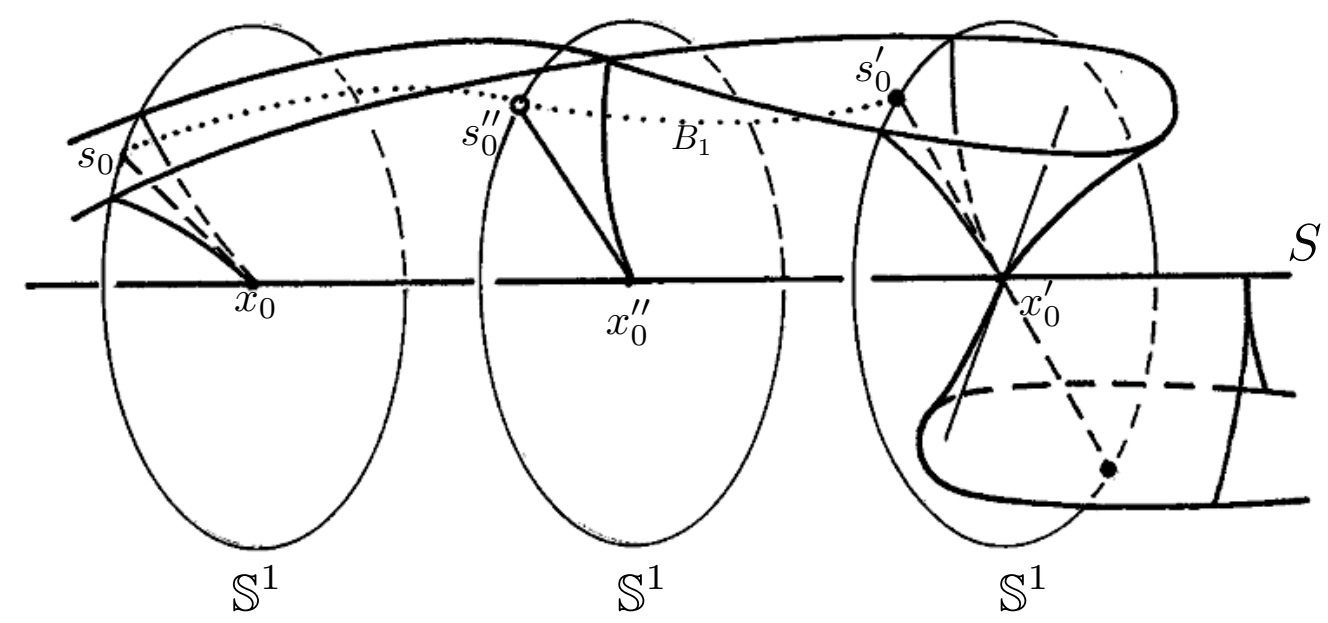

Figure 4. Schematic diagram of the discriminant $\Delta_{F}$ with cusped ridge along $0 \times S$, showing the structure close to $x_{0}, x_{0}^{\prime}, x_{0}^{\prime \prime}$ on $S$ determined by an interior point $\left(s_{0}, x_{0}\right)$, end point $\left(s_{0}^{\prime}, x_{0}^{\prime}\right)$ and intersection point $\left(s_{0}^{\prime \prime}, x_{0}^{\prime \prime}\right)$ (indicated simply by $s_{0}$ etc.) respectively.

For $(s, x)$ in a local coordinate chart around $\left(s_{0}, x_{0}\right) \in \mathbb{S}^{1} \times S$ write $(s, x)=\left(s_{0}+v, x_{0}+w\right)$ where $(v, w) \in \mathbb{R} \times \mathbb{R}$. Using (3.10) we have

$$
\alpha(\rho, v, w)=a \circ \tilde{\tau}(\rho, s, x)=\rho b(v, w)+\rho^{2} c(v, w)+O\left(\rho^{3}\right) \in \mathbb{R}^{3}
$$

where now $b(v, w)$ is shorthand for $b(s, x)$ and analogously $c(v, w)$ stands for $c(x)(s, s)$. From (4.3) we see $\alpha(\rho, v, w) \in \Delta_{H}$ when

$$
\left(\rho b_{1}+\rho^{2} c_{1}\right)^{2}+\left(\rho b_{2}+\rho^{2} c_{2}\right)^{2}\left(\rho b_{3}+\rho^{2} c_{3}\right)+O\left(\rho^{4}\right)=0
$$

which after cancelling $\rho^{2}$ reduces to

$$
\beta(\rho, v, w):=b_{1}^{2}+\left(2 b_{1} c_{1}+b_{2}^{2} b_{3}\right) \rho+d(\rho, v, w) \rho^{2}=0
$$

where $b_{i}, c_{i}$ are components of $b(v, w), c(v, w)$ and where $d(\rho, v, w)$ is a smooth real-valued function. Now we study three possibilities, illustrated in Figure 4.

(i) $\left(s_{0}, x_{0}\right)$ is an interior point: $b_{1}=0$ and $b_{2} b_{3} \neq 0$.

The IFT at $(v, w)=(0,0)$ implies that if $\tilde{\rho}:=b_{1}^{2}-\beta$ then the map $(\rho, v, w) \mapsto(\tilde{\rho}, v, w)$ is a local diffeomorphism at the origin. In $(\tilde{\rho}, v, w)$ coordinates the locus $\beta=0$ is given by $\tilde{\rho}=b_{1}^{2}$, which (since 
$(0,0)$ is a regular point of the function $b_{1}$ by hypothesis $(\mathrm{H} 1)$ of transversality to $\left.T_{2}\right)$ is a smooth surface in $\mathbb{R}^{3}$ quadratically tangent to the curve $b_{1}(v, w)=0$.

Finally, to reconstruct the geometry in the original $(\varepsilon, x)$ deformation coordinates, we apply the pinching map $\tilde{\tau}$ which takes the surface $\beta=0$ locally to a surface with a cusped ridge along $0 \times S$; the tangent direction of the cusp at $\left(0, x_{0}\right)$ is $s_{0} \in \mathbb{S}^{1}$, while as $x$ moves away from $x_{0}$ in $S$ the tangent direction $s$ of the cusp is given by the smooth curve

$$
B_{1}=\left\{(x, s) \in \mathbb{S}^{1} \times S: b_{1}(x, s)=0\right\}
$$

through $\left(s_{0}, x_{0}\right)$ in $\mathbb{S}^{1} \times S$. See Figure 4 .

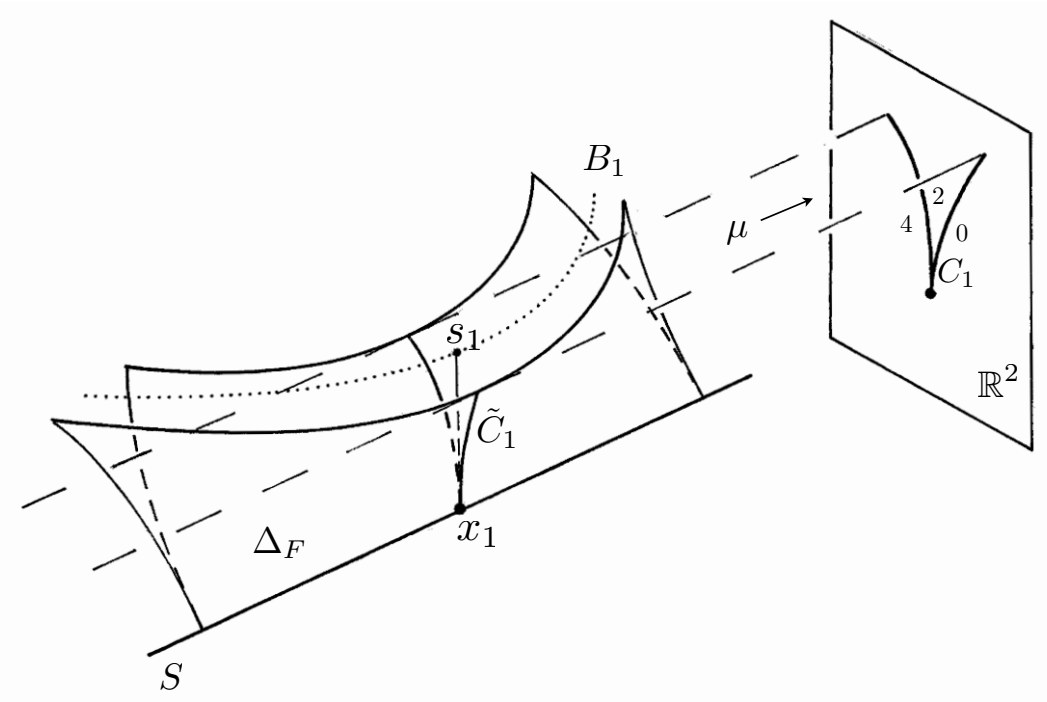

Figure 5. The projection $\mu: \Delta_{F} \rightarrow \mathbb{R}^{2}$ close to $\left(0, x_{1}\right) \in \mathbb{R}^{2} \times S$ where $\left(s_{1}, x_{1}\right)$ is an interior fold point of the curve $B_{1}$. The cusp curve $\tilde{C}_{1}$ projects to a cusp curve $C_{1}$ (the bifurcation set) in $\mathbb{R}^{2}$ tangent to the $s_{1}$ direction. The numbers $0,2,4$ indicate the number of solutions $(x, y) \in K$ to $F(\varepsilon, x, y)=0$ close to $\left(x_{1}, 0\right) \in K$ for $\varepsilon$ close to the origin in $\mathbb{R}^{2}$.

(ii) $\left(s_{0}, x_{0}\right)$ is an end point (denoted $\left.\left(s_{0}^{\prime}, x_{0}^{\prime}\right)\right): b_{1}=b_{3}=0, b_{2} \neq 0$.

From the hypothesis (H1) of transversality to $T_{1}$ we have $\frac{\partial\left(b_{1}, b_{3}\right)}{\partial(v, w)} \neq 0$ at $(0,0)$, and so the map

$$
\mathbb{R}^{2} \rightarrow \mathbb{R}^{2}:(v, w) \mapsto(\tilde{v}, \tilde{w}):=\left(b_{1}, 2 c_{1} b_{1}+b_{2}^{2} b_{3}\right)
$$

is a diffeomorphism germ at the origin. In new coordinates the function $\beta$ takes the form $\beta(\rho, \tilde{v}, \tilde{w})=$ $Q(\rho, \tilde{v}, \tilde{w})+O\left(\rho^{3}\right)$ where

$$
Q(\rho, \tilde{v}, \tilde{w})=\tilde{v}^{2}+\tilde{w} \rho+e \rho^{2}
$$

which is a nondegenerate quadratic form regardless of the value of the coefficient $e$. The locus $Q=0$ is a cone $C$ tangent to the $(\tilde{v}, \tilde{w})$-plane along the $\tilde{w}$-axis. By the Morse Lemma there is a local diffeomorphism (with derivative the identity) that takes the locus $\beta^{-1}(0)$ to the cone $C$ and, moreover, (4.7) shows that $\beta^{-1}(0)$ is quadratically tangent to the $(\tilde{v}, \tilde{w})$-plane along the smooth 
curve $\tilde{v}=b_{1}=0$. Finally, by Proposition 3.5 the pinching map $\tilde{\tau}$ takes $\beta^{-1}(0)$ to a surface with $\frac{3}{2}$-power cusped ridge along $0 \times S$ and terminating at $\left(0, x_{0}^{\prime}\right)$, reminiscent of a ship's prow: see Figure 4.

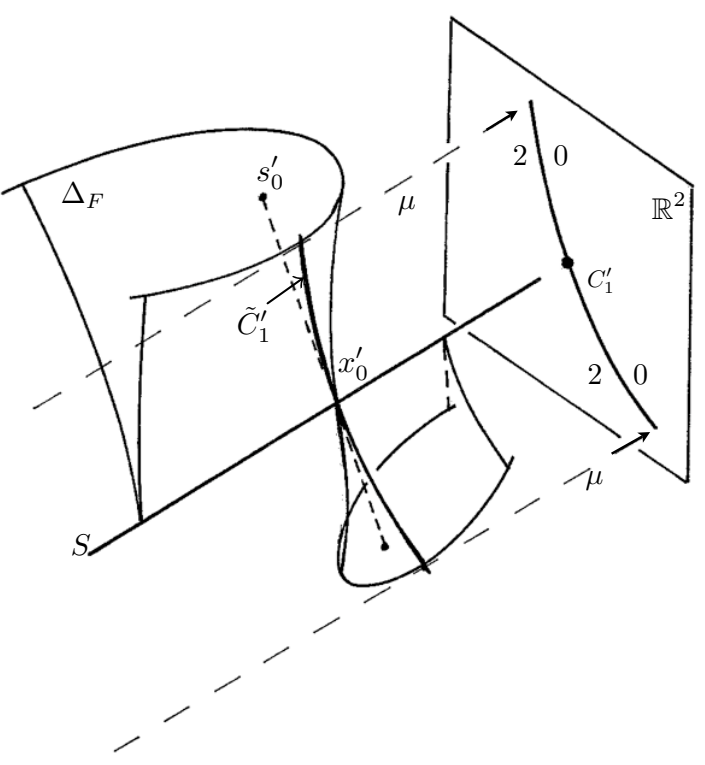

Figure 6 . The projection $\mu: \Delta_{F} \rightarrow \mathbb{R}^{2}$ close to $\left(0, x_{0}^{\prime}\right) \in \mathbb{R}^{2} \times S$ where $\left(s_{0}^{\prime}, x_{0}^{\prime}\right)$ is an end point of the curve $B_{1}$. The curve $\tilde{C}_{1}^{\prime}$ projects to a pair of arcs $C_{1}^{\prime}$ (the bifurcation set) in $\mathbb{R}^{2}$ tangent to the $s_{0}^{\prime}$ direction. The numbers 0,2 indicate the number of solutions $(x, y) \in K$ to $F(\varepsilon, x, y)=0$ close to $\left(x_{0}^{\prime}, 0\right) \in K$ for $\varepsilon$ close to the origin in $\mathbb{R}^{2}$.

(iii) $\left(s_{0}, x_{0}\right)$ is an intersection point (denoted $\left.\left(s_{0}^{\prime \prime}, x_{0}^{\prime \prime}\right)\right): b_{1}=b_{2}=0, b_{3}<0$.

By the hypothesis $(\mathrm{H} 1)$ of transversality to $T_{1}^{\prime}$ the map $(v, w) \mapsto\left(b_{1}, b_{2}\right)$ is a local diffeomorphism at $(0,0)$. From $(4.3)$ with $a_{3}<0$ we can express $\Delta_{H}$ as

$$
\left(a_{1}+\ell a_{2}\right)\left(a_{1}-\ell a_{2}\right)=0
$$

where $\ell=\sqrt{\left|a_{3}\right|}$, which on cancelling $\rho^{2}$ becomes $b_{1} \pm \ell b_{2}=0$ with $\ell=\sqrt{\rho\left|b_{3}\right|}$. Hence for fixed $\rho>0$ the expression (4.6) represents a pair of smooth curves crossing transversely at the origin, with tangent directions both tending to that of $b_{1}(v, w)=0$ as $\rho \rightarrow 0$. See Figure 4 .

4.1.3. Bifurcation geometry when $q=2$. We have described above the three generic types of local geometrical structure found in the discriminant $\Delta_{F}$, where we recall that (since $1=d<p=$ $d+k=2$ ) the discriminant is the projection into $\mathbb{R}^{2} \times S$ of the zero set:

$$
\Delta_{F}=\left\{(\varepsilon, x) \in \mathbb{R}^{2} \times S: \exists y \in \mathbb{R}, F(\varepsilon, x, y)=0\right\} .
$$

However, it is important to keep sight of the fact that in terms of the original problem $(2.1),(4.1)$ it is $(x, y)$ that are the variables while $\varepsilon$ is the perturbing parameter. Therefore to find the bifurcation behaviour of solutions $(x, y)$ as $\varepsilon$ varies in $\mathbb{R}^{2}$ we must also study the geometry of the projection 
$\mu: \Delta_{F} \rightarrow \mathbb{R}^{2}$ into the $\varepsilon$-plane and in particular its singularities. The image of the singular points (i.e. the set of singular values) is the bifurcation set; this can also be interpreted as the apparent outline (or apparent contour: see $[\mathbf{8}],[\mathbf{2 2}]$ ) of $\Delta_{F}$ when viewed along the 1-manifold $S$.

The analysis of the previous section showed that $\Delta_{F}$ has a cusped ridge along $0 \times S$ with the direction of the axis of the cusp (indicated on the unit circle $\mathbb{S}^{1} \subset \mathbb{R}^{2}$ ) given by the curve $B_{1}=$ $b_{1}^{-1}(0) \subset \mathbb{S}^{1} \times S$. (For any interior point $x \in S$ there can be only one $s \in \mathbb{S}^{1}$ with $(s, x) \in B_{1}$, so cusps may impinge on $(0, x) \in 0 \times S$ from only one direction, while at end points they do so from two opposite directions.) Therefore the singular points of $\mu \mid B_{1}: B_{1} \rightarrow \mathbb{S}^{1}$ play a key role in the structure of the bifurcation set for $F$. Accordingly, we make the following generic hypothesis:

(H2) The map $\mu \mid B_{1}: B_{1} \rightarrow \mathbb{S}^{1}$ has only fold singularities, and these are at interior points of $B_{1}$.

A fold singularity of $\mu \mid B_{1}$ is characterised in local coordinates $(v, w)$ on $\mathbb{S}^{1} \times S$ by

$$
\frac{\partial b_{1}}{\partial w}=0, \quad \frac{\partial^{2} b_{1}}{\partial w^{2}} \neq 0 \quad \text { at } \quad(v, w)=(0,0) .
$$

Following the classification in the previous section we now consider in turn the three types of point on the curve $B_{1}$.

(i) Interior points.

The first result confirms what is evident from a simple sketch. See Figure 5.

Proposition 4.1. Let $\left(s_{1}, x_{1}\right)$ be an interior point of $B_{1} \subset \mathbb{S}^{1} \times S$ such that the projection $\mu \mid B_{1}: B_{1} \rightarrow \mathbb{S}^{1}$ has a fold singularity at $\left(s_{1}, x_{1}\right)$. Then the apparent outline in $\mathbb{R}^{2}$ of a neighbourhood of $\left(0, x_{1}\right)$ in $\Delta_{F}$ viewed along $S$ is a curve $C_{1}$ with a cusp of order $\frac{3}{2}$ at the origin and with tangent direction $s_{1}$ there. The number of solutions $(x, y)$ to $F_{\varepsilon}(x, y)=0$ increases by two as $\varepsilon$ crosses each arc of $C_{1}$ in the appropriate (and same) direction of rotation about the origin.

Proof. In local coordinates $(\rho, v, w)$ the apparent outline $\Gamma_{1}$ of $\Delta_{F}^{1}$ viewed in the direction of $S$ is by definition given by

$$
\Gamma_{1}=\left\{(\rho, v) \in \mathbb{R}_{+} \times \mathbb{R}: \exists w \in S, \beta(\rho, v, w)=\frac{\partial \beta}{\partial w}(\rho, v, w)=0\right\},
$$

and we have already seen in Section 4.1.2 (i) that in suitable coordinates (in which the role of $w$ is unaffected) we may take $\beta=b_{1}^{2}-\rho$. Hence $\frac{\partial \beta}{\partial w}=2 b_{1} \frac{\partial b_{1}}{\partial w}$ which vanishes where $b_{1}=0$ (so then $\rho=0$ if $\beta=0$ ) and also where $\frac{\partial b_{1}}{\partial w}=0$. By the IFT and the fold condition (4.8), the latter locus is $\mathbb{R}_{+} \times B_{1}^{\prime}$ where $B_{1}^{\prime}$ is a smooth curve through the origin in $\mathbb{R}^{2}$ not tangent to the $w$-axis. The intersection $\Gamma_{1}$ of $\mathbb{R}_{+} \times B_{1}^{\prime}$ with $\Delta_{F}^{1}$ is a smooth curve quadratically tangent to the $(v, w)$ plane. By Proposition 3.5 the curve $\tilde{C}_{1}:=\tilde{\tau}\left(\Gamma_{1}\right)$ has a $\frac{3}{2}$-power cusp at $\left(0, x_{0}\right)$ and projects under $\mu$ to a curve $C_{1}$ with the properties described.

Note: The geometry of the curve $\tilde{C}_{1}$ in $\Delta_{F}=a^{-1}\left(\Delta_{H}\right)$ and the expression (4.2) show that as $\varepsilon$ crosses the arcs of $C_{1}$ one pair of solutions $(x, y)$ is created (or annihilated) close to $y=y_{1}$ where $y_{1}=\sqrt{-a_{3}(\varepsilon, x)}$, and the other pair close to $-y_{1}$.

(ii) End points. 
The analysis in Section 4.1.2 showed that at an end-point the surface $\Delta_{F}^{1}=\beta^{-1}(0)$ has a Morse singularity, thus a local cone structure, with the cone tangent to the $(v, w)$-plane along the curve $B_{1}$. Let $\chi=\frac{\partial \beta}{\partial w}$. From (4.7) we see that $\chi=0$ when $\rho=0$ and $b_{1}=0$, that is on the curve $B_{1}$. Differentiating the expression (4.6) with respect to $w$ we then observe that provided the generic condition

$$
\frac{\partial}{\partial w}\left(2 b_{1} c_{1}+b_{2}^{2} b_{3}\right) \neq 0
$$

holds at $(0,0)$ then $\frac{\partial \chi}{\partial \rho}(0,0) \neq 0$ and so by the IFT the locus $\chi^{-1}(0)$ locally has the form of a graph of a smooth function $\rho=\rho(v, w)$. Now $\frac{\partial \rho}{\partial w}=-\frac{\partial \chi}{\partial w} / \frac{\partial \chi}{\partial \rho}$ which is nonzero at an end point because on $B_{1}$ we have $\frac{\partial \chi}{\partial w}=2\left(\frac{\partial b_{1}}{\partial w}\right)^{2} \neq 0$ by the hypothesis (H2). Thus the smooth surface $\chi^{-1}(0) \subset \mathbb{R} \times \mathbb{R}^{2}$ cuts the $(v, w)$-plane and hence the 'cone' $\Delta_{F}^{1}$ transversely along $B_{1}$ (away from the origin) and so must cut the cone again along another smooth curve $\Gamma_{1}^{\prime}$ through the origin and transverse to the $v, w$-plane. Then $\tilde{C}_{1}^{\prime}=\tilde{\tau}\left(\Gamma_{1}^{\prime}\right)$ is a smooth curve whose image under $\mu$ is by Proposition 3.5 a $C^{1}$ arc $C_{1}^{\prime}$ through the origin in $\mathbb{R}^{2}$. See Figure 6 . To summarise:

Proposition 4.2. Let $\left(s_{0}^{\prime}, x_{0}^{\prime}\right)$ be an end point of $B_{1} \subset \mathbb{S}^{1} \times S$ and assume that $\left(H 2^{\prime}\right)$ holds. Then the apparent outline in $\mathbb{R}^{2}$ of a neighbourhood of $\left(0, x_{1}^{\prime}\right)$ in $\Delta_{F}$ viewed along $S$ is a $C^{1}$ arc $C_{1}^{\prime}$ through the origin, across each branch of which the number of solutions $(x, y)$ to $F_{\varepsilon}(x, y)=0$ increases by two, taken in the appropriate (and opposite) directions of rotation about the origin.

Remark. The arc $C_{1}^{\prime}$ is typically not the same as the 'end-point' curve $a^{-1}\left(T_{1}\right)$ which itself corresponds only to solutions with $y=0$. Thus soon after a pair of solutions is created across $C_{1}^{\prime}$ one of them will pass through $y=0$, that is through $S_{0}$. However, we are here solving the reduced equation (2.1), so the corresponding equilibria of (1.1) for $\varepsilon \neq 0$ need not lie on $S$ although they will have zero $K$-component and therefore lie in $L$.

(iii) Intersection points.

Intersection points are zeros of the map $\left(b_{1}, b_{2}\right): \mathbb{S}^{1} \times S \rightarrow \mathbb{R}^{2}$ and the zero set of $b_{1}$ is the curve $B_{1}$. We make a further generic hypothesis:

(H3) The function $b_{2}$ does not vanish at the singular points of $\mu \mid B_{1}$.

An intersection point $\left(s_{0}^{\prime \prime}, x_{0}^{\prime \prime}\right)$ corresponds to an arc of self-intersection of $\Delta_{F}$ emanating from $\left(0, x_{0}\right)$ : see Figure 4 . This arc projects under $\mu$ to an arc $C_{1}^{\prime \prime}$ from the origin in $\mathbb{R}^{2}$, and $\left(s_{0}^{\prime \prime}, x_{0}^{\prime \prime}\right)$ is a regular point of $\mu \mid B_{1}$ by the hypothesis (H3). Thus no bifurcations occur across $C_{1}^{\prime \prime}$, but two solutions of the reduced equation of opposite sign in $y$ pass through $y=0$ (that is, through $S_{0}$ ) simultaneously as $\varepsilon$ crosses $C_{1}^{\prime \prime}$, so the corresponding solutions of (1.1) pass through $L$.

We have now studied the three generic types of local bifurcation behaviour, and we assemble them into a global statement. Recall that $d=k=1$ (so the compact manifold $S$ is 1-dimensional and is degenerate with corank 1 ) and the degree $m$ of degeneracy is 2 . The perturbation parameter $\varepsilon$ lies in $\mathbb{R}^{2}$.

Let $\kappa$ denote the local involution in a neighbourhood of $S$ in $M$ that in terms of normal bundle coordinates $T S \oplus K \oplus L$ corresponds to $(x, y, z) \mapsto(x,-y, z)$. 
Theorem 4.3. Under the generic hypotheses $(\mathrm{H} 1),(\mathrm{H} 2),\left(\mathrm{H} 2^{\prime}\right),(\mathrm{H} 3)$ the bifurcation set for (1.1) consists of a finite set of $C^{1}$ arcs emanating from the origin in $\mathbb{R}^{2}$. The arcs are of three types: fold, end and intersection arcs. These correspond to the following bifurcation behaviour as $\varepsilon$ rotates about the origin in $\mathbb{R}^{2}$ in (locally) the appropriate direction.

Interior arcs. These occur in pairs with $\frac{3}{2}$-power cups at the origin. As $\varepsilon$ crosses the first arc a pair of solutions is created away from $S$ by a saddle-node (fold) bifurcation, and as $\varepsilon$ crosses the second arc a second saddle-node pair of solutions is created close to the $\kappa$-images of the first pair.

End arcs. These occur in opposite pairs forming $C^{1}$ curves through the origin, and each arc is accompanied by an arc of $a^{-1}\left(T_{1}\right)$ with the same tangent at the origin. As $\varepsilon$ crosses an end arc a pair of solutions is created away from $S$ in a saddle-node bifurcation; as $\varepsilon$ crosses the companion arc one of these solutions passes through $L$. This behaviour is repeated at the opposing end arc and with the same sense of rotation about the origin.

Intersection arcs. As $\varepsilon$ crosses an intersection arc with nonzero speed two solutions of the reduced equation with $K$-components of opposite signs pass through $L$ at the same point and with nonzero speed.

This theorem in particular recovers and provides a geometric context for the results of Hale and Taboas [32] for the case $m=2$. It also extends those results by taking account of self-intersection points of the determining curve $B_{1}$. See Figure 7, and compare Figures 1 and 2 in [32].

4.1.4. Cases $q \neq 2$. For $q=1$ (that is $\varepsilon \in \mathbb{R}$ ) the transversality hypothesis (H1) implies that the image of $b: \mathbb{S}^{0} \times S \rightarrow \mathbb{R}^{3}$ meets $T$ only at points of $T_{2}$ and that 0 is a regular value of $b_{1}: S \rightarrow \mathbb{R}$. Hence $b_{1}^{-1}(0)$ is a discrete set of points $\left\{\left( \pm 1, x_{i}\right)\right\}$. (This may be visualised in Figure 3 by taking a pair of vertical lines corresponding to fixed values $\pm s \in \mathbb{S}^{1}$.) To each $x_{i}$ with either $\varepsilon>0$ or $\varepsilon<0$ there corresponds a pair of solution branches in $\Delta_{F} \subset \mathbb{R} \times S$ emanating from $\left(0, x_{i}\right)$; the branches are such that locally $x-x_{i}$ varies as $|\varepsilon|^{\frac{3}{2}}$. In particular this describes the branching behaviour for $q=2$ when $\varepsilon$ leaves the origin along an arc not in a direction tangent to an interior, end or intersection arc as in Theorem 4.3.

For $q=3$ some interesting new geometry arises. For $x \in S$ the map $a(\cdot, x): \mathbb{R}^{3} \rightarrow \mathbb{R}^{3}$ is typically nonsingular at the origin, although generically there is a set of isolated points $\left\{x_{j}\right\} \subset S$ where the derivative $D_{j}$ of $a\left(\cdot, x_{j}\right)$ has rank 2 . For $x \notin\left\{x_{j}\right\}$ and for sufficiently small $|\varepsilon|$ the discriminant $\Delta_{F}=$ $a^{-1} \Delta_{H}$ intersects $\mathbb{R}^{3} \times\{x\}$ in diffeomorphic copy $\left\{\Delta_{H}^{x}\right\}$ of $\Delta_{H}$ (more precisely, a neighbourhood of the origin in $\Delta_{H}$, which amounts to the same thing). To capture the structure of the bifurcation set we study the map $b: \mathbb{S}^{2} \times S \rightarrow \mathbb{R}$ as in (3.9) and the geometry of $b^{-1}(T)$ where $T=T_{H}$. Writing $b_{x}(s)=b(s, x)$ we note that $B_{x}:=b_{x}^{-1}(T)=B_{x}^{0} \cup B_{x}^{1}$ where $B_{x}^{0}$ is a semicircular (open) arc $b_{x}^{-1}\left(T_{2}\right)$ of a great circle $C_{x}$ in $\mathbb{S}^{2}$ with antipodal end-points $B_{x}^{1}=b_{x}^{-1}\left(T_{1}\right)$, while $D_{F}^{1}(x):=b_{x}^{-1}\left(\Delta_{H}\right)$ is a figure eight curve on $\mathbb{S}^{2}$ passing through these end-points and with self-intersection at $b_{x}^{-1}\left(T_{1}^{\prime}\right)$. As $\rho \rightarrow 0$ the intersection $\rho \mathbb{S}^{2} \cap \Delta_{H}^{x}$ flattens to $\rho b_{x}^{-1}(T)$ at rate $\rho^{\frac{3}{2}}$.

In this setting the bifurcation set in $\mathbb{R}^{3}$, which is the set of critical values of the projection $\mu: \Delta_{F} \subset \mathbb{S}^{3} \times S \rightarrow \mathbb{R}^{3}$, is conveniently interpreted as (to first order in $\rho$ ) a cone on the envelope $E$ of the family of curves $\left\{D_{F}^{1}(x)\right\}_{x \in S}$ in $\mathbb{S}^{2}$. The curve $E$ separates points $s \in \mathbb{S}^{2}$ according to the number $n_{s}$ of points $x \in S$ with $s \in D_{F}^{1}(x)$. The generic local geometry of envelopes of smooth curves in $\mathbb{R}^{2}$ (or $\mathbb{S}^{2}$ ) is well known (see [8] for example): the envelope consists of smooth fold curves across which $n_{s}$ changes by 2 , meeting at isolated cusp points. These local configurations are stable, 


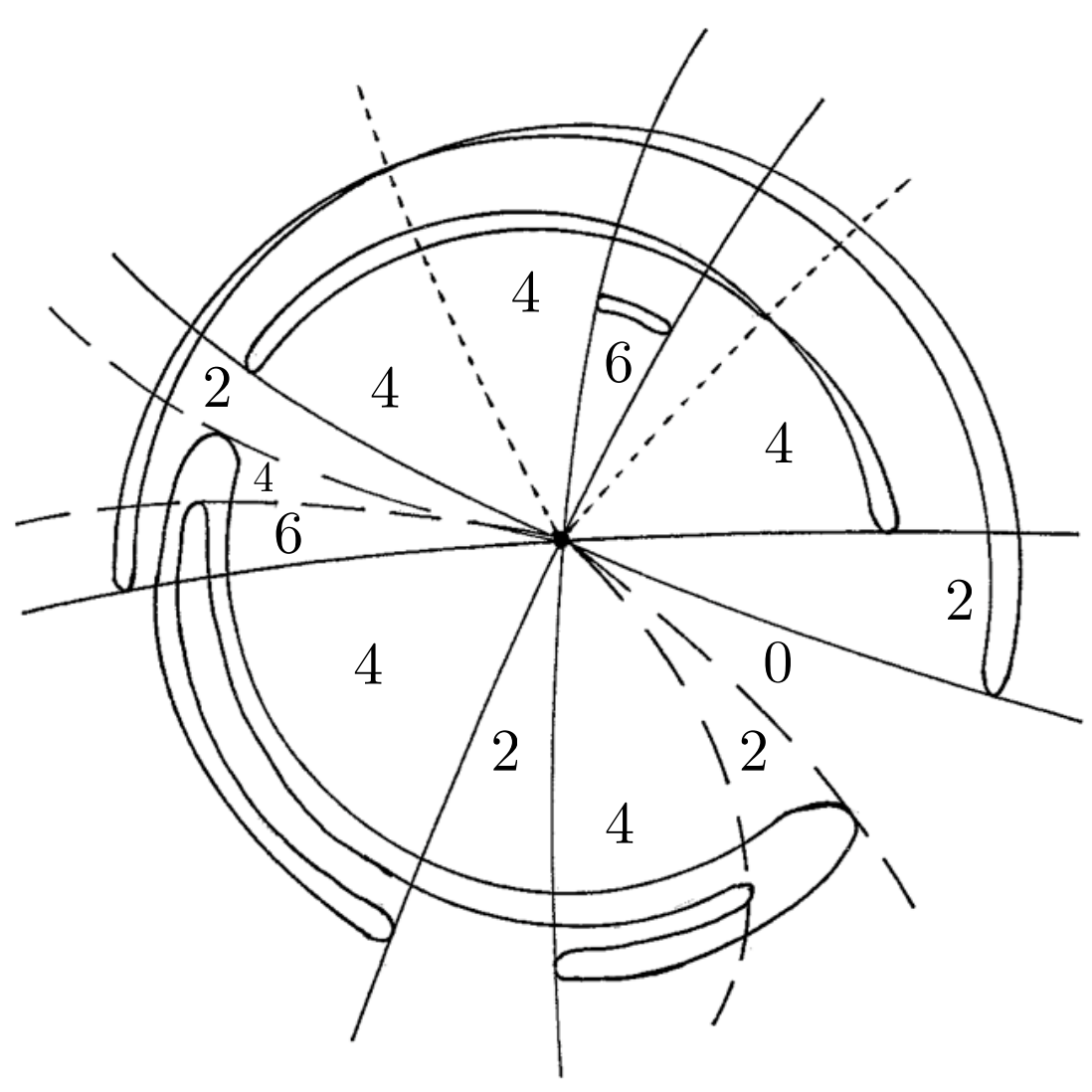

FiguRE 7. Schematic bifurcation diagram for $d=k=1, q=2$ and $m=2$ (see text), showing end arcs (unbroken lines) and fold arcs (long dashes) through the origin in the parameter space $\mathbb{R}^{2}$. Intersection arcs (short dashes) are also shown. Numbers indicate the number of solutions in each region. Note that for each opposite pair of fold arcs the two folds approach from the same side in the plane.

so that the same description applies to the envelope of the family $\left\{\rho \mathbb{S}^{2} \cap \Delta_{H}^{x}\right\}_{x \in S}$ (where higher order terms in $\rho$ are not neglected) for $\rho$ nonzero and sufficiently small.

We could leave the discussion at this point, but further useful insight is obtained by taking account of the role of $b^{-1}(T)$, which we regard (compare Section 4) as a 'first approximation' to $D_{F}^{1}(x)$. Let $C$ be the envelope of the great circles $\left\{C_{x}\right\}_{x \in S}$ and $B^{0} \subset C$ be the envelope of the semi-circular (open) $\operatorname{arcs}\left\{B_{x}^{0}\right\}_{x \in S}$. Let $B^{1}$ be the locus of end-points $\left\{B_{x}^{1}\right\}$ for $x \in S$. It is straightforward to check that if $s_{0} \in C \cap B^{1}$ and is a fold point of $C$ then $B^{1}$ is a smooth curve quadratically tangent to $C$ at $s_{0}$ where an arc of $B^{0}$ terminates: see Figure $8(\mathrm{a})$. When we now replace $B_{x}^{0}$ by the figureeight $D_{F}^{1}(x)$ then the 'approximate' envelope $B^{0} \cup B^{1}$ becomes a generic envelope configuration $E$ consisting of a fold curve $E^{0}$ close to $B^{0}$ which continues as a fold curve close to one branch of $B^{1}$, together with another fold curve $E^{1}$ close to $B^{0}$ which meets a fold curve $\tilde{E}^{1}$ close to the other branch of $B^{1}$ at a cusp point close to $s_{0}$ : see Figure $8(\mathrm{~b})$. The numbers $0,2,4$ indicate relative numbers $n_{s}$ for $s$ in each of the three complementary regions of $E$ locally. Finally, local stability implies that this configuration occurs in $\rho \mathbb{S}^{2} \cap \Delta_{F}$ for $\rho$ sufficiently small. 


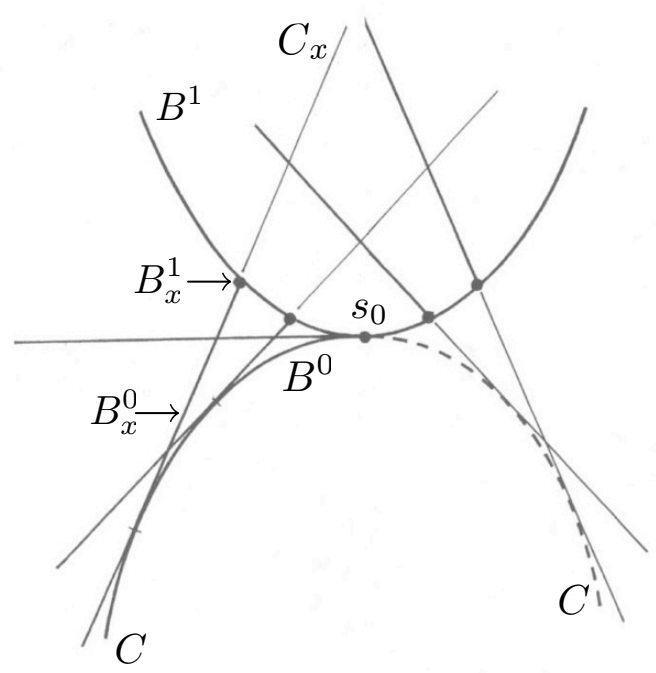

(a)



(b)

Figure 8. Envelopes in $\mathbb{S}^{2}$, sketched in the plane: (a) Local configuration of the envelope $B^{0}$ of arcs $B_{x}^{0}$ and the locus $B^{1}$ of their endpoints, (b) corresponding configuration of envelope of smooth curves $D_{F}^{1}(x)$.

Features of the earlier $q=2$ case can be seen by intersecting the above with a typical great circle $\mathbb{S}^{1}$ : we find interior points in pairs (corresponding to the pair of branches of $E$ close to $C_{0}$ ) and isolated end points (corresponding to the branches of $E$ close to $C_{1}$ ). The $q=3$ configuration shows how in the presence of a further bifurcation parameter these features generically coalesce.

Returning to the exceptional points $\left\{x_{j}\right\}$ on $S$ where rank $D_{j}=2$, let us make the generic assumption that for each $x_{j}$ neither 1-dimensional stratum $T_{1}$ or $T_{1}^{\prime}$ of $T$ lies in the range of $D_{j}$. It follows that for $x \in S$ close to $x_{j}$ the image $b_{x}\left(\mathbb{S}^{2}\right)$ is an oblate ellipsoid with 'equatorial' plane close to the range of $D_{j}$. From this and Figure 2 it can be seen that $D_{F}^{1}(x)$ has the figure-eight configuration with one loop much larger than the other. As $x$ passes through $x_{j}$ the smaller loop vanishes (the end point coalesces with the intersection point) and is re-created at the antipodal end point; the transition curve $D_{F}^{1}\left(x_{j}\right)$ in $\mathbb{S}^{2}$ has a $\frac{3}{2}$-power cusp at both its antipodal end points.

Provided $x_{j}$ does not correspond to a cusp point of the envelope $E$ the local fold structure of the envelope itself (that is, the bifurcation set in $\mathbb{S}^{2}$ ) is unaffected as $x$ passes through $x_{j}$, although the location of corresponding solutions to the bifurcation problem is controlled by this extra twist. The bifurcation structure at $x_{j}$ corresponds to one of the generic singularities of 1-parameter families of plane curves at the envelope: see [24].

For $q \geq 4$ generically for all $x \in S$ the map $a(\cdot, x): \mathbb{R}^{q} \rightarrow \mathbb{R}^{3}$ is a submersion at the origin: thus the local structure of $\Delta_{F}$ is a product $\mathbb{R}^{q-3} \times \Delta_{F}^{3}$ where $\Delta_{F}^{3}$ is as in the case $q=3$. Nevertheless, the envelope of the family $\left\{D_{F}^{1}(x)\right\}_{x \in S}$ in $\mathbb{S}^{q-1}$ may have additional singular structure, corresponding to higher-dimensional generalisations of Figure 8 which we do not investigate here. 
We now turn to cases of greater degeneracy degree $m>2$. The essential geometry, while in principle more complicated, has much in common with the case $m=2$ above.

\subsection{The case of higher degeneracy $m>2$.}

Here

$$
F(0, x, y)=h(y)=\left(0, y^{m}\right)
$$

and this germ has $\mathcal{K}$-codimension $2 m-1$ (see $[\mathbf{6}],[\mathbf{2 8}]$ ) with $\mathcal{K}$-miniversal deformation (using more systematic notation than previously) of the form

$$
H(\tilde{a}, y)=\left(a_{0}+a_{1} y+\ldots+a_{m-1} y^{m-1}, \bar{a}_{0}+\bar{a}_{1} y+\ldots+\bar{a}_{m-2} y^{m-2}+y^{m}\right)
$$

where $\tilde{a}=(a, \bar{a})=\left(a_{0}, \ldots, a_{m-1}, \bar{a}_{0}, \ldots, \bar{a}_{m-2}\right) \in \mathbb{R}^{2 m-1}$. The discriminant $\Delta_{H}$ is given by $R_{m}=0$ where $R_{m}$ is the resultant of the two components of $H(\tilde{a}, y)$ and which has the following expression as a determinant that for clarity we illustrate for $m=4$, the general pattern being analogous:

$$
R_{4}=\left|\begin{array}{ccccccc}
a_{0} & a_{1} & a_{2} & a_{3} & & & \\
& a_{0} & a_{1} & a_{2} & a_{3} & & \\
& & a_{0} & a_{1} & a_{2} & a_{3} & \\
& & & a_{0} & a_{1} & a_{2} & a_{3} \\
\bar{a}_{0} & \bar{a}_{1} & \bar{a}_{2} & 0 & 1 & & \\
& \bar{a}_{0} & \bar{a}_{1} & \bar{a}_{2} & 0 & 1 & \\
& & \bar{a}_{0} & \bar{a}_{1} & \bar{a}_{2} & 0 & 1
\end{array}\right| .
$$

Here the blanks represent zero entries.

Proposition 4.4. The resultant $R_{m}$ has the form

$$
R_{m}=a_{0}^{m}+a_{0} P_{m}+(-1)^{m} \bar{a}_{0}\left(a_{1}^{m}+a_{1} Q_{m}\right)+\bar{a}_{0}^{2}\left(a_{2}^{m}+\tilde{R}_{m}\right)
$$

where $P_{m}, Q_{m}$ are polynomials each of least degree $m$ and $\tilde{R}_{m}$ is a polynomial of degree at least $m+1$.

Here the polynomials are in the variables $a_{i}, \bar{a}_{j}$ and least degree means the degree of the lowestorder term.

Proof. The lowest-order term is $a_{0}^{m}$ from the main diagonal. Putting $a_{0}=0$ we then have $R_{m}=$ $(-1)^{m} \bar{a}_{0} R_{m-1}^{\prime}$ where $R_{m-1}^{\prime}$ is a determinant of order $2 m-2$ illustrated in the case $m=4$ as follows:

$$
R_{3}^{\prime}=\left|\begin{array}{llllll}
a_{1} & a_{2} & a_{3} & & & \\
& a_{1} & a_{2} & a_{3} & & \\
& & a_{1} & a_{2} & a_{3} & \\
& & & a_{1} & a_{2} & a_{3} \\
\bar{a}_{0} & \bar{a}_{1} & \bar{a}_{2} & 0 & 1 & \\
& \bar{a}_{0} & \bar{a}_{1} & \bar{a}_{2} & 0 & 1
\end{array}\right|
$$

The lowest-order term in $R_{m-1}^{\prime}$ is $a_{1}^{m}$, and then if $a_{1}=0$ the $\bar{a}_{0}$ in the first column is multiplied by a determinant whose lowest-order term is $(-1)^{m} a_{2}^{m}$.

Since the term of lowest order in $R_{m}$ is $a_{0}^{m}$ we see immediately: 
Corollary 4.5. The positive tangent cone $T_{H}$ lies in the hyperplane $a_{0}=0$.

As in Section 3.3 we take polar coordinates $\varepsilon=\rho s$ and write

$$
(a, \bar{a})=(a(\rho s, x), \bar{a}(\rho s, x))=\rho(b(s, x), \bar{b}(s, x))+\rho^{2}(c(s, x), \bar{c}(s, x))+O\left(\rho^{3}\right)
$$

where $(b, \bar{b}): \mathbb{S}^{q-1} \times S \rightarrow \mathbb{R}^{2 m-1}$ and likewise $(c, \bar{c})$. We then find from (4.11) that $R_{m}=\rho^{m} \bar{R}_{m}$ where

$$
\bar{R}_{m}=\bar{R}_{m}(\rho, b, \bar{b})=b_{0}^{m}+\rho\left((-1)^{m} \bar{b}_{0} b_{1}^{m}+b_{0} \tilde{P}_{m}\right)+\rho^{2} \tilde{Q}_{m+2}+O\left(\rho^{3}\right)
$$

in which $\tilde{P}_{m}, \tilde{Q}_{m+2}$ are homogeneous polynomials of degree $m, m+2$ respectively in the variables $\left(b_{i}, \bar{b}_{j}\right)$. Following the same procedure as in the case $m=2$ we first study the geometry of the set

$$
\Delta_{F}^{1}:=\left\{(\rho, s, x) \in \mathbb{R} \times \mathbb{S}^{q-1} \times S: \bar{R}_{m}(\rho, b(s, x), \bar{b}(s, x))=0\right\}
$$

since $\bar{R}_{m}(\rho, b, \bar{b})=0$ for $\rho \neq 0$ precisely when $(a, \bar{a}) \in \Delta_{H}$, and then apply the pinching map $\tilde{\tau}$ to obtain $\Delta_{F}=\tilde{\tau}\left(\Delta_{F}^{1}\right) \subset \mathbb{R}^{q} \times S$.

We shall require some transversality hypotheses analogous to (H1). Rather than formulate a general statement (which we do not need and in any case cannot carry out without specifying a stratification of $T_{H}$ ) we state the appropriate hypotheses as we come to them; they mirror those of Section 4.1.1.

Suppose $\left(s_{0}, x_{0}\right)$ satisfies $b_{0}\left(s_{0}, x_{0}\right)=0$, and take a local coordinate chart $(s, x)=\left(s_{0}+v, x_{0}+w\right)$ on $\mathbb{S}^{q-1} \times S$ as before. As in the $m=2$ analysis we look at three cases.

(i) $\quad \bar{b}_{0} b_{1} \neq 0$ at $\left(s_{0}, x_{0}\right)$.

Here by the IFT the locus $\bar{R}_{m}=0$ locally has the form

$$
\rho=k_{0} b_{0}^{m}+l_{m+1}, \quad k_{0} \neq 0,
$$

where $b_{0}=b_{0}\left(s_{0}+v, x_{0}+w\right)$ and where $l_{m+1}$ denotes terms of degree at least $m+1$ in $v, w$. We now make the generic hypothesis

$\left(\mathrm{H} 1_{m}(i)\right) \quad\left(s_{0}, x_{0}\right)$ is a regular point of the function $b_{0}$.

Here $\Delta_{F}^{1}$ is locally a smooth hypersurface in $\mathbb{R} \times \mathbb{S}^{q-1} \times S$ having contact of order $m$ with $0 \times$ $\mathbb{S}^{q-1} \times S$ along the codimension-1 submanifold

$$
B_{0}:=\left\{(s, x) \in \mathbb{S}^{q-1} \times S: b_{0}(s, x)=0\right\} .
$$

Applying the pinching map $\tilde{\tau}$, remembering that $\tau(\rho,-s)=\tau(-\rho, s)$ and using Proposition 3.5 we obtain $\Delta_{F}$ locally as a $C^{1}$ hypersurface in $\mathbb{R}^{q} \times S$ containing $0 \times S$ and having contact of order $(m+1) / m$ along $0 \times S$ with the 'direction locus' in $\mathbb{R}^{q} \times S$ determined by $B_{0}$. Thus for $q=2$, when $m=2$ the discriminant $\Delta_{F}$ has a $\frac{3}{2}$-power cusped ridge along $0 \times S$ as already seen in Section 4.1.2, while for $m=3$ it is a $C^{1}$ submanifold of $\mathbb{R}^{2} \times S$ passing through $0 \times S$.

(ii) $\quad \bar{b}_{0}=0, b_{1} \neq 0$ at $\left(s_{0}, x_{0}\right)$.

Here we make the next generic hypothesis:

$\left(\mathrm{H} 1_{m}(i i)\right) \quad\left(s_{0}, x_{0}\right)$ is a regular zero of the map $\left(b_{0}, \bar{b}_{0}\right): \mathbb{S}^{q-1} \times S \rightarrow \mathbb{R}^{2}$. 
First take $q=2$. The transversality hypothesis $\left(\mathrm{H} 1_{m}(i i)\right)$ means that we can regard the pair of functions $\left(b_{0}, \bar{b}_{0}\right)$ as local coordinates on $\mathbb{S}^{1} \times S$ close to $\left(s_{0}, x_{0}\right)$. We can then write $\bar{R}_{m}$ in the form

$$
p^{m}+\rho(\bar{p}+c \rho+O(2))
$$

where $(p, \bar{p}):=\left(b_{0},\left(-b_{1}\right)^{m} \bar{b}_{0}+b_{0} \tilde{P}_{m}\right)$ and $c$ is a constant. The quadratic terms are nondegenerate regardless of $c$, so by the Splitting Lemma (see $[\mathbf{1 7}],[\mathbf{2 2}, \S 4.5],[\mathbf{3 7}]$ ) we can suppose the coordinates are such that $\Delta_{F}^{1}$ has the form

$$
p^{m}+\rho(\bar{p}+c \rho)=0 .
$$

The description of this locus depends on whether $m$ is even or odd.

The case when $m$ is even is relatively easy. If $m=2 l$ then we write $t=p^{l}$ and the left hand side of (4.16) becomes a nondegenerate quadratic form in $(\rho, t, \bar{p})$ : the zero set is a cone with vertex at the origin, tangent to the $(t, \bar{p})$-plane and with the $\bar{p}$-axis as a generator. On reverting to $(\rho, p, \bar{p})$ coordinates the cone retains these features, although the contact with the plane $\rho=0$ is now of order $m$ rather than quadratic. Applying $\tilde{\tau}$ then gives $\Delta_{F}$ with a 'ship's prow' geometry at $\left(0, x_{0}\right)$ in $\mathbb{R}^{q} \times S$ as in Section 4.1.2 (see Figure 4) but with cusp contact of order $(m+1) / m$.

The case when $m$ is odd is a little harder to describe pictorially. Some insight is gained by regarding (4.16) as the equation of a curve in the $(\rho, p)$-plane for each fixed $\bar{p}$. As $\bar{p}$ increases through zero, the order- $m$ tangency of the locus with the plane $\rho=0$ along the $\bar{p}$-axis reverses its orientation as a 'bulge' passes through the origin from positive to negative $\rho$ where as before we interpret $(-\rho) s$ as $\rho(-s)$ when $\rho<0$. A 3-dimensional representation is attempted in Figure 9.



Figure 9 . The solution locus $\Delta_{F}^{1}$ given by $\bar{R}_{\frac{m}{2}}=0$ for $m=3, q=2$ at a point where $b_{0}=\bar{b}_{0}=0$. Here the picture is constructed by taking $\bar{R}_{3}=p^{3}+\bar{p} \rho+\rho^{2}$ where $p, \bar{p}$ are the coordinates $p=b_{0}, \quad \bar{p}=(-1)^{m} b_{1}^{m} \bar{b}_{0}+b_{0} \tilde{P}_{m}$ (see text).

For the general case when $q>2$ the local structure of $\Delta_{F}$ is the cartesian product of the above with $\mathbb{R}^{q-2}$. 
(iii) $\quad \bar{b}_{0} \neq 0, b_{1}=0$ at $\left(s_{0}, x_{0}\right)$.

Substituting $a_{0}=a_{1}=0$ into the expression (4.11) for $R_{m}$ we obtain $R_{m}=\bar{a}_{0}^{2} D_{m}$ where $D_{m}$ is a polynomial whose term of lowest order is $a_{2}^{m}$. Therefore with $q=2$ we make the further generic hypothesis

$\left(\mathrm{H} 1_{m}(i i i)\right) \quad b_{2}\left(s_{0}, x_{0}\right) \neq 0$.

It then follows that $(\varepsilon, x) \notin \Delta_{F}$ for sufficiently small $\left|x-x_{0}\right|$ and $|\varepsilon|$. Thus there is generically no analogue of intersection points in $\Delta_{F}$ when $m>2$.

When $q>2$ there exist generically points at which $b_{0}=b_{1}=b_{2}=0$, which we do not investigate further here.

4.2.1. The bifurcation set for degeneracy degree $m>2$. Having found the local form of the discriminant $\Delta_{F} \subset \mathbb{R}^{q} \times S$ under generic assumptions, we are now in a position to describe the generic structure of the bifurcation set, that is the apparent outline of $\Delta_{F}$ viewed along $S$. Recall that $\Delta_{F}$ is the locus $\bar{R}_{m}=0$ where $\bar{R}_{m}$ is given by (4.13) with $(a, \bar{a})=\rho(b, \bar{b})$. Again we work first with $q=2$.

Taking local coordinates $(s, x)=\left(s_{0}+v, x_{0}+w\right)$ on $\mathbb{S}^{1} \times S$ we seek common solutions to the equations

$$
\bar{R}_{m}=0, \quad \frac{\partial}{\partial w} \bar{R}_{m}=0
$$

As before, we consider two cases.

(i) $\bar{b}_{0} b_{1} \neq 0$ at $\left(s_{0}, x_{0}\right)$.

Here the argument parallels that of Section 4.1.3. We make the generic hypothesis

$\left(\mathrm{H} 2_{m}(i)\right)$ The projection $\mu \mid B_{0}: B_{0} \rightarrow \mathbb{S}^{1}$ has only fold singularities.

If $\left(s_{0}, x_{0}\right)$ is a regular point of $\mu \mid B_{0}$ then no local bifurcations occur for any solution branches in directions sufficiently close to $s_{0}$. If $\left(s_{0}, x_{0}\right)$ is a fold point of $\mu \mid B_{0}$ then from (4.14) and following Section 4.1.3 we find that the apparent outline of $\Delta_{F}^{1}$ in the direction of $S$ is locally a smooth curve $\Gamma_{0}$ through $\left(0, s_{0}\right)$ and having contact of order $m$ with the $(v, w)$-plane, so that in particular when $m$ is odd it has only one branch for $\rho>0$ while for $m$ even it has two. Applying the pinching map $\tilde{\tau}$ to $\Delta_{F}^{1}$ and projection $\mu$ gives a curve $C_{0}$ through the origin in parameter space $\mathbb{R}^{2}$ having contact of order $(m+1) / m$ with its tangent direction $s_{0}$. The number of solutions $(x, y)$ changes by two as each branch of $C_{0}$ is crossed.

(ii) $\quad \bar{b}_{0}=0, b_{1} \neq 0$ at $\left(s_{0}, x_{0}\right)$.

Here we make the further generic hypothesis

$$
\left(\mathrm{H} 2_{m}(i i)\right) \quad \frac{\partial b_{0}}{\partial x}\left(\left(-b_{1}\right)^{m} \frac{\partial \bar{b}_{0}}{\partial x}+\tilde{P}_{m} \frac{\partial b_{0}}{\partial x}\right) \neq 0 \text { at }\left(s_{0}, x_{0}\right) .
$$

In terms of coordinates $(p, \bar{p})$ used above, this simply states that $\xi:=\frac{\partial p}{\partial x}$ and $\eta:=\frac{\partial \bar{p}}{\partial x}$ are both nonzero at $(p, \bar{p})=(0,0)$. From (4.15) and again disregarding the higher order terms as in (4.16) 
we have

$$
\frac{\partial \bar{R}_{m}}{\partial x}=m p^{m-1} \xi+\rho \eta
$$

so that $\frac{\partial \bar{R}_{m}}{\partial x}=0$ is solved as $\rho=\tilde{\rho}(p)=\lambda p^{m-1}$ with constant $\lambda=-m \xi / \eta \neq 0$ at $(0,0)$. Substituting into (4.16) and deleting the factor $p^{m-1}$ we obtain

$$
p+\lambda \bar{p}+O\left(p^{m-1}\right)=0
$$

which represents a smooth curve $B_{0}^{\prime}$ through the origin in the $(p, \bar{p})$-plane, transverse to both axes and not tangent to $(\xi, \eta)$ since $m \neq 1$. Projecting the graph of $\tilde{\rho} \mid B_{0}^{\prime}$ to a plane orthogonal to $(\xi, \eta, 0)$ then gives the apparent outline of $\Delta_{F}^{1}$ viewed along $S$ : this curve $C_{0}^{1}$ has one branch for $\rho>0$ and one for $\rho<0$ when $m$ is even and two branches for $\rho>0$ when $m$ is odd. After applying the pinching map $\tau$ the curve $C_{0}^{1}$ becomes a bifurcation curve $C_{0}$ having contact of order $(m+1) / m$ with its tangent line at the origin. For $m$ even the number of solutions changes locally from 0 to 2 as the curve is crossed; for $m$ odd it changes from 1 to 3 and back again in a hysteresis loop as the two branches are successively crossed. See Figure 10.

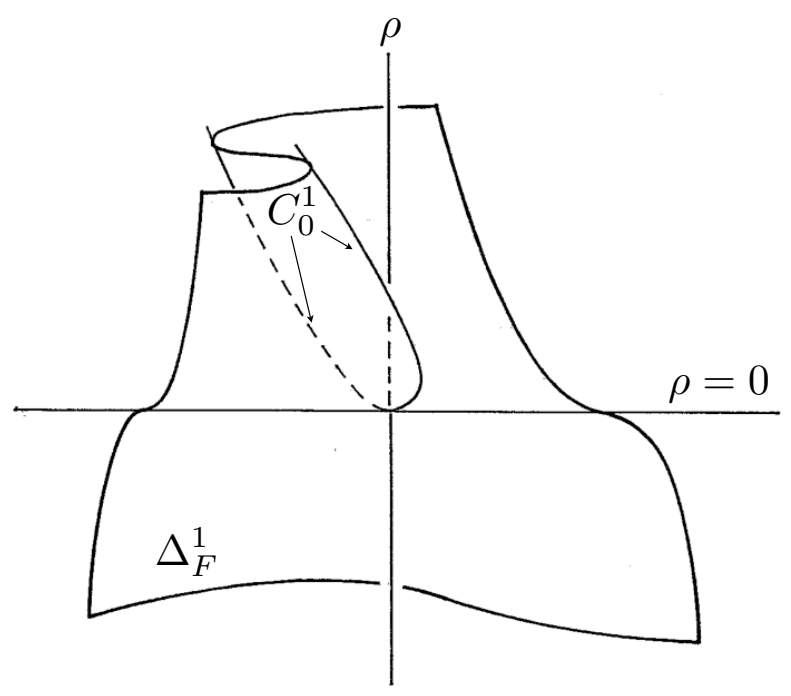

Figure 10. The discriminant $\Delta_{F}^{1}$ for $m=3$ viewed in a typical direction not parallel to $p, \bar{p}$ axes: apparent outline $C_{0}^{1}$ (bifurcation curve) in $\mathbb{R}^{2}$ becomes a cusped curve at the origin after applying the pinching map $\tau$.

We summarise the bifurcation results in the following theorem. Recall that we are in the same dimensional setting as Theorem 4.3 but now with degeneracy degree $m>2$.

THEOREM 4.6. Under the generic hypotheses $\left(\mathrm{H} 1_{m}(i)-(i i i)\right)$ and $\left(\mathrm{H} 2_{m}(i)-(\right.$ ii $\left.)\right)$ the bifurcation set for (1.1) consists of a finite set of $C^{1}$ arcs emanating from the origin in $\mathbb{R}^{2}$, each making contact of order $\frac{m+1}{m}$ with its tangent ray at the origin. The arcs are of two types: fold and end arcs, corresponding to the following bifurcation behaviour as $\varepsilon$ rotates about the origin in $\mathbb{R}^{2}$ in (locally) the appropriate direction: 
Fold arcs. These occur in pairs with a common tangent at the origin, emanating in the same or opposite directions from the origin according as $m$ is even or odd respectively. In the even case, as $\varepsilon$ crosses the first arc in the appropriate direction a pair of solutions is created away from $S$ by a saddle-node (fold) bifurcation, and as $\varepsilon$ crosses the second arc a second saddle-node pair of solutions is created close to the $\kappa$-images of the first pair. In the odd case a saddle-node bifurcation occurs across each arc.

End arcs $(m$ even). These occur in pairs with a common tangent at the origin, emanating in opposite directions from the origin. As $\varepsilon$ crosses an end arc in the appropriate direction a pair of solutions is created away from $S$ in a saddle-node bifurcation; the two directions are in opposing senses of rotation about the origin.

Hysteresis arcs $(m$ odd). These occur in pairs with a common tangent at the origin, emanating in the same direction from the origin. As $\varepsilon$ crosses the first arc in the appropriate direction a saddlenode bifurcation creates a pair of solutions, one of which coalesces with an existing solution (via a hysteresis loop) in a saddle-node bifurcation across the second arc.

This elaborates on and gives a geometric setting for Theorem 2.1B of [32]. See Figure 11, and compare with Figures 3 and 4 of $[\mathbf{3 2}]$. Note that the interpretation regarding solutions $(x, y)$ passing through $y=0$ does not hold in the general case $m>2$ since $\bar{a}_{0}=0$ does not imply $a_{0}=0$ for points on $\Delta_{H}$.

Remark. These results on the structure of $\Delta_{F}$ and the form of the expression (4.9) show that for $\varepsilon \in \mathbb{R}$ and $d=k=1$ the generic solution branches are of the form $(\varepsilon, x, y) \sim\left(t^{m}, t^{m+1}, t\right)$. This applies also in the case $m=1$ (normal nondegeneracy) where $y$ represents a coordinate transverse to $S$ and the nonzero solution value $y$ varies linearly with $\varepsilon$.

\subsection{The variational case}

As noted in Section 2.1, when the original vector field $F_{\varepsilon}$ is the gradient of a smooth real-valued function $f_{\varepsilon}$ then the reduced vector field $\tilde{F}_{\varepsilon}$ may be regarded as the gradient of a real-valued function $\tilde{f}_{\varepsilon}$. Therefore in this setting the bifurcations of equilibria are controlled not as in Section 3 by $\mathcal{K}$-versal deformation of the map germ

$$
\tilde{F}_{0}\left(x_{0}, \cdot\right): \mathbb{R}^{k} \rightarrow \mathbb{R}^{d} \times \mathbb{R}^{k}
$$

at $0 \in \mathbb{R}^{k}$ but rather by an $\mathcal{R}$-versal deformation of the function germ

$$
\tilde{f}_{0}\left(x_{0}, \cdot\right): \mathbb{R}^{k} \rightarrow \mathbb{R}
$$

at $0 \in \mathbb{R}^{k}$ where $\tilde{F}_{0}=\operatorname{grad} \tilde{f}_{0}$. In the case of uniform normal degeneracy of $S$ the germ $\tilde{f}_{0}$ is independent of $x_{0} \in S$.

To study the bifurcation geometry we follow the formal procedure of Section 3.2 but now in addition to the simplification in dealing with functions rather than vector fields there is an added twist: the gradient of a deformation of $\tilde{f}_{0}\left(x_{0}, \cdot\right)$ will involve derivatives with respect to the 'parameter' $x$ as well as with respect to the variable $y$.

Assume we are in the uniform case, and write $\tilde{f}_{0}(x, y)=\tilde{f}_{0}(y)$. If $\tilde{f}_{0}$ has an $r$-parameter $\mathcal{R}$-versal deformation of the form

$$
a_{1} v_{1}(y)+\ldots+a_{r} v_{r}(y)+\tilde{f}_{0}(y)
$$




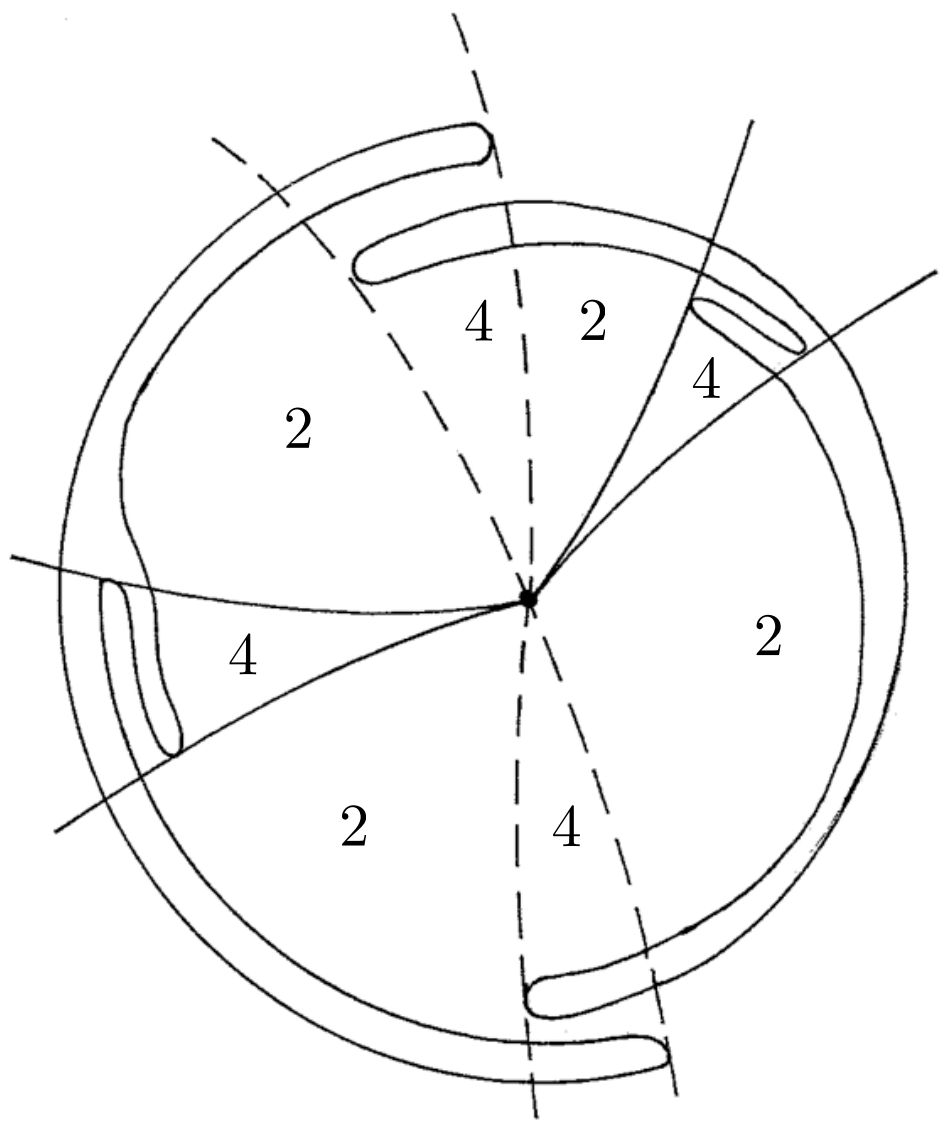

FiguRE 11. Schematic bifurcation diagram for $d=k=1, q=2$ and $m$ odd (see text), showing hysteresis arcs (unbroken lines) and fold arcs (dashed lines) through the origin in parameter space $\mathbb{R}^{2}$. Numbers indicate the number of solutions in each region. Note that for each opposite pair of fold arcs the two folds approach from opposite sides in the plane.

then as in Corollary 3.4 we express $\tilde{f}_{\varepsilon}$ as

$$
\tilde{f}_{\varepsilon}=a_{1}(\varepsilon, x) v_{1}(\tilde{y})+\ldots+a_{r}(\varepsilon, x) v_{r}(\tilde{y})+\tilde{f}_{0}(\tilde{y})
$$

and now (after dropping the tilde) we find $F_{\varepsilon}=\left(\partial_{x} f_{\varepsilon}, \partial_{y} f_{\varepsilon}\right)$ where

$$
\begin{aligned}
& \partial_{x} f_{\varepsilon}=a_{1}^{\prime}(\varepsilon, x) v_{1}(y)+\ldots+a_{r}^{\prime}(\varepsilon, x) v_{r}(y) \\
& \partial_{y} f_{\varepsilon}=a_{1}(\varepsilon, x) v_{1}^{\prime}(y)+\ldots+a_{r}(\varepsilon, x) v_{r}^{\prime}(y)+F_{0}(y)
\end{aligned}
$$

and we write $a_{i}^{\prime}, v_{j}^{\prime}$ for $\operatorname{grad}_{x} a_{i}, \operatorname{grad}_{y} v_{j}$ respectively. The condition for $(\varepsilon, x)$ to belong to the discriminant $\Delta_{F}$ is an algebraic condition on the 1-jet (with respect to $x$ ) of the map $a=\left(a_{1}, \ldots, a_{r}\right): \mathbb{R}^{q} \times S \rightarrow \mathbb{R}^{r}$. We illustrate this with a simple but non-trivial example that exhibits both the similarities with and the differences from the general geometric formalism of previous sections. 
Example. Let $k=1$ and consider the bifurcation problem $F_{\varepsilon}=0$ where $F_{\varepsilon}=\operatorname{grad} f_{\varepsilon}$ with

$$
f_{\varepsilon}(x, y)=\varepsilon g(\varepsilon, x, y)+y^{m} .
$$

An $\mathcal{R}$-versal deformation of the function germ $y^{m}$ is

$$
a_{0}+a_{1} y+\ldots+a_{m-2} y^{m-2}+y^{m}
$$

(see $[\mathbf{6}],[\mathbf{2 8}]$ ) and so for $m=3$ we can write

$$
f_{\varepsilon}(x, y)=a_{0}(\varepsilon, x)+a_{1}(\varepsilon, x) y+y^{3}
$$

and we have

$$
\begin{aligned}
& \partial_{x} f_{\varepsilon}(x, y)=a_{0}^{\prime}(\varepsilon, x)+a_{1}^{\prime}(\varepsilon, x) y \\
& \partial_{y} f_{\varepsilon}(x, y)=a_{1}(\varepsilon, x)+3 y^{2} .
\end{aligned}
$$

The condition for $(\varepsilon, x)$ to belong to the discriminant $\Delta_{F}$ in the case $d=1$ (compare (4.3)) is then

$$
3\left(a_{0}^{\prime}(\varepsilon, x)\right)^{2}+\left(a_{1}^{\prime}(\varepsilon, x)\right)^{2} a_{1}(\varepsilon, x)=0 .
$$

Following the geometric analysis of Section 4 we write $\varepsilon=\rho s$ in polar coordinates and

$$
a(\rho s, x)=\rho b(s, x)+O\left(\rho^{2}\right)
$$

and find that the conditions for $b(\varepsilon, x)$ to lie in the positive tangent cone $T$ are

$$
b_{0}^{\prime}(x)=0, \quad b_{1}(s, x) \leq 0 .
$$

where $b_{0}^{\prime}=\operatorname{grad}_{x} b_{0}$. For simplicity of description we take as usual the case $q=2$ so $(s, x) \in \mathbb{S}^{1} \times S$. Generically the locus

$$
B_{0}^{\prime}=\left\{(s, x) \in \mathbb{S}^{1} \times S: b_{0}^{\prime}(s, x)=0\right\}
$$

is a collection of smooth curves (codimension-1 manifold) such that the singularities of the projection $\mu \mid B_{0}^{\prime}: B_{0}^{\prime} \rightarrow \mathbb{S}^{1}$ are isolated folds. There are at least two of these on each compact connected component of $S$. (For $q \leq 4$ the singularities of $\mu$ will generically be folds, cusps $(q=3,4)$ or swallowtails $(q=4)$, while for $q>4$ singularities of corank $>1$ can appear. See [29, Ch.VII, $\S 6]$ for example.) Likewise the locus

$$
B_{1}=\left\{(s, x) \in \mathbb{S}^{1} \times S: b_{1}(s, x)=0\right\}
$$

is generically a smooth curve (codimension- 1 manifold) which intersects $B_{0}^{\prime}$ transversely at points which are not singular points of the projection $\mu \mid B_{0}^{\prime}$. The analysis proceeds as in Sections 4.1.2 and 4.1.3 noting that now end-points are given by $B_{0}^{\prime} \cap B_{1}$ while intersection points are the critical points of $b_{1} \mid B_{0}$. Thus the generic bifurcation geometry turns out to be the same as in Theorem 4.3 for the (non-variational) case $m=2$ although the interpretation of key features is here tied to the variational structure.

For the general case $f_{0}(y)=y^{m}$ we define the 1-jet map

$$
j^{1} a: \mathbb{R}^{q} \times S \rightarrow J^{1}\left(S, \mathbb{R}^{m-1}\right):(\varepsilon, x) \mapsto\left(x ; a_{0}, a_{1}, a_{2}, \ldots, a_{m-2} ; a_{0}^{\prime}, a_{1}^{\prime}, \ldots, a_{m-2}^{\prime}\right)
$$

where $a_{i}=a_{i}(\varepsilon, x)$ and $a_{i}^{\prime}=\operatorname{grad}_{x} a_{i}$ for $i=0,1, \ldots, m-2$. Taking local coordinates on $S$ we consider the map

$$
h: \mathbb{R} \rightarrow \mathbb{R}^{d+1}: y \mapsto\left(0, \ldots, 0, m y^{m-1}\right)
$$


which has $\mathcal{K}$-codimension $e:=(d+1)(m-1)-1$ (see [28]) and a $\mathcal{K}$-miniversal deformation $H$ with discriminant $\Delta_{H} \subset \mathbb{R}^{e}$. Locally $J^{1}\left(S, \mathbb{R}^{m-1}\right) \cong \mathbb{R}^{d} \times \mathbb{R}^{m-1} \times \mathbb{R}^{d(m-1)}=\mathbb{R}^{d} \times \mathbb{R}^{e+1}$ and we let $\Delta_{f}=\left(\pi \circ j_{1} a\right)^{-1} \Delta_{H}$ where $\pi$ is projection of $J^{1}\left(S, \mathbb{R}^{m-1}\right)$ onto the last $e$ coordinates (that is, omitting $x$ and $\left.a_{0}\right)$. We then consider the related map

$$
\tilde{b}: \mathbb{S}^{q-1} \times S \rightarrow \mathbb{R}^{e}:(s, x) \mapsto\left(b_{1}, b_{2}, \ldots, b_{m-2}, b_{0}^{\prime}, b_{1}^{\prime}, \ldots, b_{m-2}^{\prime}\right)
$$

with $b: \mathbb{S}^{q-1} \times S \rightarrow \mathbb{R}^{m-1}$ defined as in (4.19). Under suitable non-degeneracy hypotheses on $\tilde{b}$ which, using the Thom jet transversality theorem (see $[\mathbf{6}],[\mathbf{2 2}],[\mathbf{2 8}],[\mathbf{2 9}]$ ), we expect to be generically satisfied by the map $f_{\varepsilon}$, local geometry of the discriminant $\Delta_{f}$ and its projection to the parameter space $\mathbb{R}^{q}$ (giving the bifurcation structure) can in principle be determined - at least for small values of $q$ and $m$. We leave details to the enthusiastic reader.

\subsection{Possible further reduction}

In the general case a further reduction is technically possible, using the IFT one more time. For simplicity of illustration take $q=1$ so that $\varepsilon \in \mathbb{R}$.

Given $x \in S$, write $\tilde{g}(x)=\widetilde{\mathcal{F}}(0, x, 0)$ where $\widetilde{\mathcal{F}}: \mathbb{R} \times U \rightarrow \mathbb{R}^{d+k}$ is the deformation map as in (2.5) with $(x, y) \in U$. We make the following assumption, which holds generically as $\operatorname{dim} S=d<d+k$ :

(A4) The image of $\tilde{g}: S \rightarrow \mathbb{R}^{d+k}$ avoids the origin in $\mathbb{R}^{d+k}$.

This means that given $x_{0} \in S$ there is at least one component $\tilde{g}_{i}$ of $\tilde{g}$ that does not vanish at $\left(x_{0}, 0\right)$ when $\varepsilon=0$. By the IFT the $i^{\text {th }}$ component of $\tilde{F}_{\varepsilon}=0$ can then be solved close to $u=\left(x_{0}, 0\right)$ in the form $\varepsilon=\varepsilon_{i}(x, y)$. Substituting this into the other components of $\tilde{F}_{\varepsilon}$ yields $d+k-1$ equations to be solved for $(x, y)$ close to $(0,0) \in \mathbb{R}^{d} \times \mathbb{R}^{k}$. The solution set can be viewed as the zero set for a $d$-parameter family of smooth map germs $\mathbb{R}^{k} \rightarrow \mathbb{R}^{d+k-1}$, where techniques of versal deformation still apply, now in one lower dimension in parameter space and target.

For example, if $d=1$ then we have a 1-parameter deformation of germs $\mathbb{R}^{k} \rightarrow \mathbb{R}^{k}$ about which much is known for small $k$ : see $[\mathbf{4 0}]$ for the case $k=2$. If $d=2$ then we are dealing with germs $\mathbb{R}^{k} \rightarrow \mathbb{R}^{k+1}$ : see $[\mathbf{3 5}]$ for the case $k=2$ here.

In this formal reduction of the problem, however, the controlling role of $\varepsilon$ has been sacrificed, and it is not clear that there is practical benefit to understanding the bifurcation structure. We do not pursue this approach further.

\subsection{Nontrivial bundles}

In the case of nontrivial bundles $N S, T S, K$ etc, where the same coordinates $(x, y)$ cannot be used on the whole of $U \subset K$, the deformation theory must be re-expressed in terms of jet bundles. Given smooth vector bundles $D, E$ over $S$ and a family of bundle maps $F_{\varepsilon}: D \rightarrow E$ with $\varepsilon \in \mathbb{R}^{q}$, the generic structure of $F_{\varepsilon}$ is expressed in terms of transversality of the $r$-jet extension

$$
j^{r} F: \mathbb{R}^{q} \times D \rightarrow J^{r}(D, E):(\varepsilon, u) \mapsto j^{r} F_{\varepsilon}(u)
$$

to suitable stratifications of the $r$-jet bundle $J^{r}(D, E)$ and for sufficiently large $r$ (cf. [5]). In our context this theory has to be applied to the reduced vector field $\tilde{F}_{\varepsilon}: K \rightarrow T S \oplus P$. The geometry of the zero set is captured in local coordinates using $\mathcal{K}$-versal deformation theory as we have done, 
but global aspects are dictated by the geometry of the bundles. It would be interesting to explore this further even in the simplest case $d=k=1$ with $K$ both the trivial and nontrivial $\mathbb{R}$-bundle over a circle.

\section{Local branching analysis in the 1-parameter case with corank 1.}

It is one thing to understand generic structure of the bifurcation set, but another to recognise the specific branching geometry in particular cases. In this section we focus on the latter. We return to the key case of corank $k=1$ with one bifurcation parameter $(q=1)$, and take advantage of the fact that a singular function of one variable $y \in \mathbb{R}$ is dominated by the first nonvanishing term of its Taylor series in order to classify the local branching behaviour of $\tilde{F}_{\varepsilon}$ in a systematic algebraic way (bottom up) that complements the generic geometrical approach (top down) that we have described so far.

Recall from (2.5) that we have in local coordinates

$$
\tilde{F}(\varepsilon, u)=\tilde{F}_{0}(u)+\varepsilon \tilde{\mathcal{F}}(\varepsilon, u)
$$

where $u=(x, y) \in S \times \mathbb{R}$ and $\varepsilon \in \mathbb{R}$. Each component of $\tilde{F}_{0}(u)$ has a Taylor series in $y$ with coefficients depending smoothly on $x \in S$. We do not immediately assume here that the normal degeneracy is uniform, but we suppose some control on degeneracy by making the following assumption:

(A5) Each component of $\tilde{F}_{0}(u)$ has nonzero Taylor series in $y$ at every point $x \in S$.

In other words, the normal degeneracy in the problem is of finite order everywhere on $S$. This is not essential in all that follows, for it will be clear how to amend the discussion to allow infinite degeneracy in one component. We leave aside, however, the complications of infinite degeneracy in more than one component.

\subsection{Necessary conditions for branching}

From (2.3) and (2.4) the $i^{\text {th }}$ component of $\tilde{F}(\varepsilon, u)$ can be written in the form

$$
\tilde{F}_{i}(\varepsilon, u)=\varepsilon g_{i}(\varepsilon, u)+y^{m_{i}} r_{i}(u)
$$

for some integer $m_{i} \geq 2$ and a smooth function $r_{i}: U \rightarrow \mathbb{R}$; we suppose that $r_{i}(\cdot, 0)$ is not identically zero, otherwise we would increase the exponent $m_{i}$. Let $x_{0} \in S$ and number the coordinates so that at $x_{0}$

$$
m_{1} \leq m_{2} \leq \ldots \leq m_{d+1}
$$

Proposition 5.1. Necessary conditions for $x_{0} \in S$ to be a branch point are that for $1 \leq i<$ $j \leq d+1$

$$
\begin{array}{lll}
r_{i} g_{j}=0 \quad \text { when } & m_{i}<m_{j} \\
d_{i j}=0 \quad \text { when } & m_{i}=m_{j}
\end{array}
$$

for all $1 \leq i<j \leq d+1$, where $g_{i}=g_{i}\left(0, x_{0}, 0\right), r_{j}=r_{j}\left(x_{0}, 0\right)$ and $d_{i j}:=r_{i} g_{j}-r_{j} g_{i}$. 
Proof. From (5.2) we see that

$$
\tilde{F}_{i}(\varepsilon, u) g_{j}(\varepsilon, u)-\tilde{F}_{j}(\varepsilon, u) g_{i}(\varepsilon, u)=y^{m_{i}} r_{i}(u) g_{j}(\varepsilon, u)-y^{m_{j}} r_{j}(u) g_{i}(\varepsilon, u) .
$$

Dividing through by $y^{m_{i}}$ and letting $(\varepsilon, x, y) \rightarrow\left(0, x_{0}, 0\right)$ immediately gives the results.

The conditions (5.3) are not all independent. Given that the assumption (A5) holds, choose $x_{0} \in S$ and let

$$
n=n\left(x_{0}\right)=\max \left\{i: g_{i} \neq 0\right\} .
$$

Then the conditions (5.3) reduce to

$$
\begin{array}{lll}
\text { (a) } \quad r_{i}=0 \quad \text { when } \quad m_{i}>m_{n} \\
\text { (b) } d_{i j}=0 \quad \text { when } & m_{i}=m_{j}=m_{n} .
\end{array}
$$

Let $J_{n}=\left\{j: m_{j}=m_{n}\right\}$ : then $J_{n}$ is an integer interval $[l, p]$ say, with $l \leq n \leq p$. In (5.5) the number of conditions $(a)$ is $l-1$ and the number of independent conditions $(b)$ is $p-l$. Thus (5.5) represents $p-1$ conditions, which together with

$$
g_{n+1}=\ldots=g_{d+1}=0
$$

give a total of $d-n+p$ conditions on $x_{0} \in S$. Hence we have the following result.

Corollary 5.2. Generically no points $x_{0} \in S$ satisfy the conditions (5.5) when $n<p$, and there is a set of isolated points $x_{0} \in S$ which satisfy (5.5) when $n=p$.

In the uniform case the results can be expressed more simply. Each $r_{i}$ is nonzero everywhere on $S$ and the exponents $m_{i}$ do not depend on $x \in S$, so if branching is to occur at any $x_{0} \in S$ we must have $n=p$ (since if $n<i \leq p$ then $d_{n i}=0$ implies $r_{i}=0$ ) and also $l=1$ (else $r_{j}=0$ for $j<l$ ). Hence we obtain in this context the neater result:

Corollary 5.3. In the uniform case a necessary condition for $x_{0} \in S$ to be a branch point is that $g_{[n]}\left(x_{0}\right)=0$ where

$$
g_{[n]}:=\left(d_{2}, \ldots, d_{n}, g_{n+1}, \ldots, g_{d+1}\right): S \rightarrow \mathbb{R}^{d}
$$

in which $d_{j}$ denotes $d_{1 j}$ for $j=n, \ldots, d$ and $n=\max \left\{i: m_{i}=m_{1}\right\}$.

Of course, if $m_{1}<m_{2}$ (so $m_{1}<m_{j}$ for all $j>1$ ) then $g_{[n]}$ contains no $d_{j}$ terms and the result is even more straightforward :

Corollary 5.4. If $m_{1}<m_{2}$ then branch points $x_{0} \in S$ must be zeros of the map

$$
\bar{g}:=\left(g_{2}, \ldots, g_{d+1}\right): S \rightarrow \mathbb{R}^{d} .
$$

Generically $0 \in \mathbb{R}^{d}$ will be a regular value of $\bar{g}$ and the zeros of $\bar{g}$ will be a discrete subset of $S$. In any given context there may be analytical or topological tools (for example, index theory) available to locate or count them. 
The previous results gave necessary conditions for solution branches to emanate from $\left(0, x_{0}, 0\right) \in$ $\mathbb{R} \times U \subset \mathbb{R} \times S \times \mathbb{R}$. We now turn to sufficient conditions.

\subsection{Sufficient conditions for branching}

Let $x_{0} \in S$ satisfy the necessary conditions (5.5) for branching. With the notation of Section 5.1 since $g_{n} \neq 0$ at $\left(0, x_{0}, 0\right)$ we can use the IFT to solve the equation $\tilde{F}_{n}(\varepsilon, u)=0$ from $(5.2)$ in the form

$$
\varepsilon=\varepsilon_{n}(u)=y^{m_{n}} \delta_{n}(u)
$$

where $\delta_{n}$ is a smooth function on a neighbourhood of $\left(x_{0}, 0\right)$ in $U \subset K$. Substituting this into each component $\tilde{F}_{i}$ with $i \neq n$ and dividing through by appropriate powers of $y$ we see that finding a solution branch amounts to finding solutions $u=(x, y)$ close to $\left(x_{0}, 0\right)$ to the set of equations:

$$
\begin{aligned}
& \delta_{n}(u) g_{i}\left(\varepsilon_{n}(u), u\right)+y^{m_{i}-m_{n}} r_{i}(u)=0, \quad i>n \\
& d_{n j}\left(\varepsilon_{n}(u), u\right)=0, \quad l \leq j<n \\
& y^{m_{n}-m_{j}} \delta_{n}(u) g_{j}\left(\varepsilon_{n}(u), u\right)+r_{j}(u)=0, \quad j<l .
\end{aligned}
$$

Again by the IFT, these equations will have a smooth solution branch in the form $x=x(y)$ (that is, transverse to $S_{0}$ in $U$ ) if and only if the Jacobian matrix of the map

$$
S \rightarrow \mathbb{R}^{d}: x \mapsto\left(r_{1}, \ldots, r_{l-1}, d_{l}, \ldots, d_{n-1}, \delta_{n} g_{n+1}, \ldots, \delta_{n} g_{d+1}\right)
$$

(there are no $d_{j}$ terms if $n=l$ ) is nonsingular at $x_{0}$. Now suppose

$$
r_{n}:=r_{n}\left(x_{0}, 0\right) \neq 0
$$

which is satisfied automatically in the uniform case. Then solving $\tilde{F}_{n}(\varepsilon, u)=0$ shows that $\delta_{n}\left(x_{0}, 0\right) \neq 0$. Differentiating $\delta_{n} g_{i}$ at $\left(0, x_{0}, 0\right)$ gives

$$
\partial_{x}\left(\delta_{n} g_{i}\right)\left(0, x_{0}, 0\right)=\delta_{n}\left(x_{0}, 0\right) \partial_{x} g_{i}\left(0, x_{0}, 0\right)
$$

for $i>n$ as $g_{i}\left(x_{0}, 0\right)=0$. Therefore, since $\delta_{n}\left(x_{0}, 0\right) \neq 0$, the Jacobian condition above can be restated as follows.

Proposition 5.5. Given that the necessary conditions (5.3) are satisfied at $x_{0} \in S$ and (5.8) also holds, a sufficient condition for the existence of a solution branch emanating from $\left(x_{0}, 0\right)$ and transverse to $S_{0}$ in $K$ is that $x_{0}$ be a regular zero of the map

$$
g_{l, n}:=\left(r_{1}, \ldots, r_{l-1}, d_{l}, \ldots, d_{n-1}, g_{n+1}, \ldots, g_{d+1}\right): S \rightarrow \mathbb{R}^{d}
$$

the $r$ and $d$ terms being absent when $n=1$.

In the uniform case (where all $r_{j} \neq 0$ ) the expression is again much simpler: since $m_{1}=\ldots=m_{n}$ and there are no $r_{j}$ terms we have:

COROLlary 5.6. In the uniform case, a sufficient condition for $x_{0} \in S$ to be a branch point is that $x_{0}$ be a regular zero of the map $g_{[n]}: S \rightarrow \mathbb{R}^{d}$.

As before, note that if $m_{1}<m_{2}$ then $g_{[n]}$ becomes the map $\bar{g}:\left(g_{2}, \ldots, g_{d+1}\right): S \rightarrow \mathbb{R}^{d}$. 
Remark. We can regard the normally nondegenerate case as an extension of the above: here $m_{1}=1$ and all other $m_{i}>1$, and so regular zeros of $\bar{g}$ are branch points.

Remark. In general if a zero $x_{0}$ of $g_{l, n}$ is not a regular zero then progress can still be made by standard bifurcation techniques. For example, if the Jacobian matrix at $x_{0}$ has rank $d-1$ then a further Lyapunov-Schmidt reduction leads to a single bifurcation function of one variable, whose zero set can be determined locally from the quadratic terms if nondegenerate or from higher order terms by standard singularity-theory methods otherwise.

\subsection{Examples}

We illustrate the preceding formal descriptions by some simple examples.

Example $1 \quad(\operatorname{dim} S=1) \quad \tilde{F}_{0}(x, y)=\left(r_{1}(x, y) y^{2}, r_{2}(x, y) y^{3}\right)$.

The equations to solve for the zero set of the deformation $\tilde{F}_{\varepsilon}(x, y)$ are

$$
\begin{aligned}
& \varepsilon g_{1}(\varepsilon, x, y)+r_{1}(x, y) y^{2}=0 \\
& \varepsilon g_{2}(\varepsilon, x, y)+r_{2}(x, y) y^{3}=0 .
\end{aligned}
$$

From Proposition 5.1 a necessary condition for $x_{0} \in S$ to be a branch point is that $x_{0}$ be a zero of the function

$$
x \mapsto r_{1}(x, 0) g_{2}(0, x, 0)
$$

and a sufficient condition is that $x_{0}$ be a simple zero.

Example 2 $(\operatorname{dim} S=1) \quad \tilde{F}_{0}(x, y)=\left(r_{1}(x, y) y^{2}, r_{2}(x, y) y^{2}\right)$.

With $\left(g_{1}, g_{2}\right)$ as in (5.9) a necessary condition now is that $x_{0}$ be a zero of the function

$$
d_{12}: x \mapsto g_{1}(0, x, 0) r_{2}(x, 0)-g_{2}(0, x, 0) r_{1}(x, 0)
$$

and a sufficient condition is that $x_{0}$ be a simple zero.

In the uniform case the functions $r_{1}, r_{2}$ are nonzero constants and (as we have seen in general) the necessary and sufficient conditions are conditions on $g_{1}, g_{2}$ alone. Generically $g_{2}$ (Example 1 ) or $d_{12}$ (Example 2) will have a discrete set of zeros on $S$, all of them simple.

Example $3 \quad(\operatorname{dim} S=2) \quad \tilde{F}_{0}(x, y)=\left(r_{1}(x, y) y^{2}, r_{2}(x, y) y^{3}, r_{3}(x, y) y^{4}\right)$.

Here with the usual notation we see that necessary conditions for $x_{*} \in S$ to be a branch point are that $r_{1} g_{2}=r_{1} g_{3}=r_{2} g_{3}=0$ at $\left(x_{*}, 0\right) \in S \times \mathbb{R}$, or in other words that $x_{*}$ is a zero either of $\left(r_{1} g_{2}, g_{3}\right)$ or of $\left(r_{1}, r_{2}\right)$, these maps $S \rightarrow \mathbb{R}^{2}$ being evaluated with $y=0$. In the uniform case this reduces to $x_{*}$ being a zero of $\left(g_{2}, g_{3}\right)$; then being a regular zero is a sufficient condition for $x_{*}$ to be a branch point.

We leave to the reader the extension of this example to the cases when some components of $\tilde{F}_{0}$ have leading terms with equal powers of $y$.

\subsection{The variational case}

We now consider how this local branching analysis applies in the variational case. For simplicity we take $q=k=1$. 
Following the preceding sections and dropping the tilde notation let $f: \mathbb{R} \times U \rightarrow \mathbb{R}$ be a smooth function such that $d f_{0}(u)=0$ and $d^{2} f_{0}(u)=0$ for all $u=(x, 0) \in S_{0}$, where $f_{\varepsilon}(u)=f(\varepsilon, u)$. Assuming the non-flatness condition (A5) we write $f$ in the form

$$
f(\varepsilon, x, y)=\varepsilon g(\varepsilon, x, y)+y^{m} r(x, y)
$$

with $m \geq 3$ and $r(\cdot, 0)$ not identically zero on $S$; in the uniform case $r(\cdot, 0) \neq 0$ on $S$. Critical points of $f_{\varepsilon}: U \rightarrow \mathbb{R}$ occur where

$$
\left\{\begin{array}{l}
\varepsilon \partial_{x} g(\varepsilon, x, y)+y^{m} \partial_{x} r(x, y)=0 \in \mathbb{R}^{d} \\
\varepsilon \partial_{y} g(\varepsilon, x, y)+m y^{m-1} r(x, y)+y^{m} \partial_{y} r(x, y)=0 \in \mathbb{R} .
\end{array}\right.
$$

Proposition 5.7. A necessary condition for $x_{0} \in S$ to be a branch point is

$$
r\left(x_{0}, 0\right) \partial_{x} g\left(0, x_{0}, 0\right)=0 \in \mathbb{R}^{d} .
$$

Proof. The argument of Proposition 5.1 leads (after cancelling $y^{m-1}$ ) to the equation

$$
m r(x, y) \partial_{x} g(\varepsilon, x, y)+y\left[\partial_{x} g(\varepsilon, x, y) \partial_{y} r(x, y)-\partial_{y} g(\varepsilon, x, y) \partial_{x} r(x, y)\right]=0
$$

and as $(\varepsilon, x, y) \rightarrow\left(0, x_{0}, 0\right)$ we obtain $(5.11)$.

COROLlary 5.8. In the uniform case all branch points are critical points of $g(0, \cdot, 0): S_{0} \rightarrow \mathbb{R}$.

Proposition 5.9. If (5.11) holds for $x_{0} \in S$, then a sufficient condition for $x_{0}$ to be a branch point is provided by either of the following conditions at $x_{0}$ :

(i) $\partial r / \partial x_{n-1} \neq 0$ and $x_{0}$ is a regular zero of the map

$$
x \mapsto\left(m r, e_{2}, \ldots, e_{n-1}, h_{n}, \ldots, h_{d}\right): S \rightarrow \mathbb{R}^{d}
$$

where $e_{j}:=-\frac{\partial g}{\partial y} \frac{\partial r}{\partial x_{j-1}}$ and $h_{i}=\frac{\partial g}{\partial x_{i}}$

(ii) $r \neq 0$ and $x_{0}$ is a nondegenerate critical point of $g(0, \cdot, 0)$.

Proof. If $\partial_{x} g\left(0, x_{0}, 0\right) \neq 0$ then $r\left(x_{0}, 0\right)=0$ by (5.11). Condition (5.8) here is $\partial r / \partial x_{n-1} \neq 0$, where (with the notation of Section 5.1) we have $J_{n}=[2, d+1]$ and (i) follows as in Proposition 5.5: note that $d_{j}$ reduces to $e_{j}$ since $r=0$.

If $\partial_{x} g\left(0, x_{0}, 0\right)=0$ then again from Proposition 5.5 with (5.8) we obtain (ii).

Corollary 5.10. In the uniform case $r \neq 0$ and it is only the condition (ii) that applies.

Here, just as in the normally nondegenerate case, we can use topological tools such as LyusternikSchnirelmann category or Morse Theory in order to give a lower bounds for the number of branch points: see $[\mathbf{3}, \mathbf{9}, \mathbf{3 9}]$. Observe that this global result characterising the branch points is the same as in the normally nondegenerate case. However, the branching behaviour itself is different: recall the Remark following Theorem 4.6. 


\section{Applications}

Here we outline some examples of problems where the techniques presented in this paper can be applied.

\subsection{Periodic orbits}

A standard technique for finding periodic orbits (of period $T$ ) for a vector field $X_{0}$ is to consider them as zeros of the nonlinear operator

$$
F_{0}:=\frac{d}{d t}-X_{0}
$$

defined on a suitable Banach space $\mathcal{H}$ of $T$-periodic functions. There is a natural action of the circle group $\mathbb{S}^{1}$ on $\mathcal{H}$ given by time-translation modulo $T$, and if $F_{0}(x)=0$ then also $F_{0}\left(x_{s}\right)=0$ for all $s \in \mathbb{S}^{1}=\mathbb{R} \bmod T$, where $x_{s}(t)$ denotes $x(s+t)$. Thus each zero of $F_{0}$ is automatically a member of an $\mathbb{S}^{1}$-orbit $S$ of zeros. When this orbit is normally nondegenerate the problem essentially reduces to a problem on $S$ and techniques that are now standard $([\mathbf{3}, \mathbf{1 3}, \mathbf{1 6}, \mathbf{2 0}, \mathbf{3 1}, \mathbf{4 2}])$ can be applied to study bifurcations of zeros of $F_{0}$ under perturbations of $X_{0}$. The first authors apparently to attempt a systematic approach to normal degeneracy in this context were Hale and Taboas [32] who used analytic methods to study a differential equation of the form

$$
\ddot{x}+g(x)=\varepsilon_{1} h(t) \dot{x}+\varepsilon_{2} f(t) \in \mathbb{R}
$$

close to a degenerate $T$-periodic solution of $\ddot{x}+g(x)=0$, where $h$ and $f$ are $T$-periodic functions. As indicated in Section 4.1.3 our Theorem 4.3 recovers their results and sets them in a geometric context.

\subsection{Hamiltonian systems}

In the case of Hamiltonian systems, where periodic orbits may typically lie in flow-invariant tori, each zero of $F_{0}$ will lie in a larger-dimensional manifold (torus) of zeros. We expect normal nondegeneracy of this manifold within each energy level, but for discrete values of the Hamiltonian function (energy) we may find normal degeneracy. This appears both in the study of the Poincaré map for the periodic orbits and equivalently in the study of manifolds of critical points of the Jacobi metric.

To illustrate this in a relatively simple situation where the periodic orbits are isolated in each energy level, consider a Hamiltonian system in $\mathbb{R}^{2}$ with a smooth Hamiltonian of the form

$$
H(q, p)=K(p)+V(q)
$$

where $(q, p) \in T^{*} \mathbb{R}^{2} \cong \mathbb{R}^{2} \times \mathbb{R}^{2}$, and where $K(p)=|p|^{2} / 2$. Up to parametrisation, a periodic orbit $q(\cdot)$ for the system is a critical point of the Jacobi metric (see $[\mathbf{1}$, Sect.3.7,3.8] for example)

$$
\mathcal{J}[q]=\int_{0}^{1}(E-V(q(s))) K(\dot{q}(s)) d s
$$

which has natural time-translation $\mathbb{S}^{1}$-invariance as a smooth function on $\mathcal{H}=H^{1}\left([0,1], \mathbb{R}^{2}\right)$; here $E$ is a constant greater than $V(q)$ throughout the region of $\mathbb{R}^{2}$ under consideration. 
Assume that the system is rotationally symmetric about the origin, so that $V(q)=V(r)$ with $r=|q|$. The Euler-Lagrange equation derived from $\mathcal{J}$ is

$$
\frac{d}{d t}\left[\dot{q}(E-V(r)]+\frac{|\dot{q}|^{2}}{2} \frac{1}{r} V^{\prime}(r) q(t)=0\right.
$$

where ' denotes $\frac{\partial}{\partial r}$. Now let $q_{0}(t)$ be a uniform circular orbit written as $q_{0}(t)=r_{0} e(t)$ where $e(t) \in \mathbb{R}^{2}$ is a unit vector. Substituting $q_{0}(t)$ into (6.1) and using the identity $\ddot{e}(t)=-|\dot{e}(t)|^{2} e(t)$ gives

$$
\left(E-V\left(r_{0}\right)\right)=\frac{r_{0}}{2} V^{\prime}\left(r_{0}\right)
$$

as a necessary and sufficient condition for $q_{0}(t)$ to be a periodic orbit for the system. Note that circular periodic orbits of fixed radius form a manifold $S_{E}$ in $\mathcal{H}$ that is a copy of $\mathbb{S}^{1}$ :

$$
S_{E}=\left\{q_{s} \in \mathcal{H}: q_{s}(t)=R_{s} q_{0}(t)\right\}
$$

where $R_{s} \in S O(2)$ denotes rotation through angle $s \in[0,2 \pi)$.

To ascertain normal (non)degeneracy we next study the second derivative $D^{2} \mathcal{J}\left[q_{0}\right]$ which is defined on on the space $\mathcal{H}$ and corresponds to a densely defined self-adjoint operator on $L^{2}\left([0,1], \mathbb{R}^{2}\right)$. We show in the Appendix (Section 7.1) that given (6.2) the kernel of $D^{2} \mathcal{J}\left[q_{0}\right]$ has dimension 2 when

$$
r_{0} V^{\prime \prime}\left(r_{0}\right)=\left(2 n^{2}-9\right) V^{\prime}\left(r_{0}\right)
$$

for some $n \in \mathbb{Z}$, and has dimension 1 (corresponding to $T_{q_{0}} S$ ) otherwise. Note that condition (6.3) can be read in terms of discrete values $\left\{E_{n}\right\}$ of the energy $E$ :

$$
E_{n}=V\left(r_{0}\right)+\frac{r_{0}^{2}}{2\left(2 n^{2}-9\right)} V^{\prime \prime}\left(r_{0}\right) .
$$

For energy values $E_{n}$ the corresponding $S_{E_{n}}$ is a normally degenerate manifold of points representing periodic orbits.

A potential $V(q)$ for which all this can be explicitly verified is the so-called Mexican hat:

$$
V(q)=-\frac{\lambda^{2}}{2}|q|^{2}+\frac{1}{4}|q|^{4} .
$$

The above methods allow us to detect the presence of periodic orbits that persist after perturbing $V$ in the annular Hill's region given by $\left\{q \in \mathbb{R}^{2}: V(q) \leq E\right\}$ where $-\frac{1}{4} \lambda^{4}<E<0$. Setting $E=E_{n}$ and fixing $u \in K_{q_{0}}$ where $K_{q_{0}}$ is a complement to $T_{q_{0}} S_{E_{n}}$ in ker $D^{2} \mathcal{J}\left[q_{0}\right]$ it is not difficult to check that the Taylor expansion about $q_{0}$ of the Jacobi metric after Lyapunov-Schmidt reduction reads

$$
\mathcal{J}(y):=\mathcal{J}\left[q_{0}+y u\right]=\mathcal{J}\left[q_{0}\right]+\mathcal{J}_{0} y^{4}+O\left(y^{5}\right)
$$

where $\mathcal{J}_{0}=\frac{1}{4 !} D^{4} \mathcal{J}\left[q_{0}\right](u, u, u, u)$. This places us in the context of Section 4.3 with $d=k=1$ and $m=4$.

An example of bifurcation can be seen by considering a potential

$$
V_{\varepsilon}(q)=V(q)+\varepsilon_{1} \phi_{1}(q)+\varepsilon_{2} \phi_{2}(q)
$$

where $\phi_{i}(q)=\phi_{i}\left(q_{1}, q_{2}\right)$ is a smooth function, $i=1,2$. Up to degree 4 in $y$ the reduced Jacobi metric is now

$$
\mathcal{J}_{\varepsilon}\left[q_{0}+y u\right]=\mathcal{J}\left[q_{0}\right]+\mathcal{J}_{0} y^{4}+\varepsilon_{1} \Phi_{1}\left[q_{0}, y\right]+\varepsilon_{2} \Phi_{2}\left[q_{0}, y\right]
$$


where

$$
\Phi_{i}\left[q_{0}, y\right]=-\int_{0}^{1} d t\left\|\dot{q}_{0}(t)\right\|^{2}\left(\phi_{i}\left(q_{0}(t)+y u(t)\right)\right.
$$

for $i=1,2$. Now let $q_{0}(t)=\left(r_{0} \cos (\omega t+x), r_{0} \sin (\omega t+x)\right)$ be an element of $S_{E_{n}}$ parametrised by $x \in S^{1}$. The expression (6.6) reduces to

$$
\mathcal{J}(\varepsilon, x, y):=\mathcal{J}\left[q_{0}\right]+\mathcal{J}_{0} y^{4}+\varepsilon_{1} g_{1}(x, y)+\varepsilon_{2} g_{2}(x, y)
$$

up to degree 4 in $y$, where

$$
g_{i}(x, y)=\Phi_{i}\left[q_{0}, y\right]
$$

for $i=1,2$. Following the Example given in Section 4.3 but now with $m=4$ we see that the bifurcation geometry is determined by the geometry of the curves $B_{0}^{\prime}$ and $B_{1}$ in $\mathbb{S}^{1} \times S$ given by $b_{0}^{\prime}(s, x)=0$ and $b_{1}(s, x)=0$ respectively, where $b_{0}, b_{1}$ are derived from the perturbation $g$ as in Section 4.2 with $m=4$.

Remark. The case of a single parameter $\varepsilon($ so $q=1)$ can be interpreted in this context as a fixed choice of $s \in \mathbb{S}^{1}$. Here the function $g(0, \cdot, 0): S_{0} \rightarrow \mathbb{R}$ of Section 5.4 corresponds to the function $b_{0}(s, \cdot)$ of Sections 4.3 and 4.2. The necessary condition for $x_{0}$ to be a branch point as given by Proposition 5.7 corresponds to the statement that the curve $B_{0}^{\prime}$ intersects the circle $\{s\} \times S$ at $\left(s, x_{0}\right)$, and the sufficient condition given by Proposition 5.9(ii) corresponds to the statement that it does so transversely. Since every smooth function on a circle has at least two critical points there will be at least two such intersections, and in any case an even number if they are transverse.

\subsection{Steady states in chemical reaction networks}

In kinetic models for chemical reaction networks the time evolution of a vector $x(t)=$ $\left(x_{1}(t), \ldots, x_{n}(t)\right)$ of concentrations of $n$ chemical species involved in $r$ reactions is given by

$$
\frac{d x}{d t}=F(x, k):=B \nu(x, k)
$$

where $B$ is an $n \times r$ matrix (stochiometric coefficients) and

$$
\nu: \mathbb{R}^{n} \times \mathbb{R}^{c} \rightarrow \mathbb{R}^{r}
$$

is a smooth map, often in fact a polynomial map. A review of this type of system can be found in [23]. The components of $k \in \mathbb{R}^{c}$ are the reaction constants. For given $k$ the stationary states of (6.7) are thus given by the set

$$
S_{k}:=\left\{x \in \mathbb{R}^{n}: \nu_{k}(x) \in \operatorname{ker}(B)\right\}
$$

where $\nu_{k}(x)=\nu(x, k)$.

In applications, one is interested in studying steady states of perturbed systems of the form

$$
F_{\varepsilon}(x):=B \nu(x, k)+\varepsilon \phi(\varepsilon, x)
$$

for $\varepsilon \in \mathbb{R}^{q}$ where $\phi(\varepsilon, x)$ is smooth (not necessarily polynomial) function. It is therefore appropriate to apply our analysis to systems of this type.

Let $R \subset \mathbb{R}^{n}$ denote the range of $B$ and let $\pi: \mathbb{R}^{n} \rightarrow R$ be a projection onto $R$. Write $\tilde{B}=\pi \circ B$ : $\mathbb{R}^{r} \rightarrow R$. If $0 \in R$ is a regular value of $\tilde{B} \circ \nu_{k}$ (that is, the map $\nu_{k}$ is transverse to $\operatorname{ker} B$ ) then $S_{k}$ 
is a smooth submanifold of $\mathbb{R}^{n}$ with codimension equal to the dimension of $R$ (the rank of $B$ ); moreover, $\operatorname{dim} S_{k}=\operatorname{dim} \operatorname{ker} D F(x)$ for $x \in S_{k}$ so the manifold $S_{k}$ is normally nondegenerate.

If 0 is not a regular value of $\tilde{B} \circ \nu_{k}$ it means that there is at least one point $x \in S_{k}$ at which $\nu_{k}$ fails to be transverse to $\operatorname{ker} B$. Situations can arise where this occurs simultaneously at all points of $S_{k}$ and we have uniform normal degeneracy. We present now a simple example of this class of system.

Consider a set of reactions of the form

$$
X_{1}+X_{2} \stackrel{1}{\rightarrow} X_{3}, \quad X_{3} \stackrel{1}{\rightarrow} X_{1}+X_{2}, \quad \emptyset \stackrel{v\left(\left[X_{1}\right]\right)}{\longrightarrow} X_{2}
$$

where the first two reaction rates are fixed to 1 and the third is a function $v$ of the concentration $\left[X_{1}\right]$ of $X_{1}$. The third reaction is interpreted as an external input whose rate is a function of $\left[X_{1}\right]$. Now let $\left[X_{i}\right]=x_{i}$ denote the $i$-th concentration, so $x=\left(x_{1}, x_{2}, x_{3}\right) \in \mathbb{R}_{+}^{3}$ (all components non-negative) and the dynamic equations read

$$
\begin{aligned}
& \dot{x}_{1}(t)=-x_{1}(t) x_{2}(t)+x_{3}(t) \\
& \dot{x}_{2}(t)=-x_{1}(t) x_{2}(t)+x_{3}(t)+v\left(x_{1}(t)\right) \\
& \dot{x}_{3}(t)=x_{1}(t) x_{2}(t)-x_{3}(t)
\end{aligned}
$$

where here we take $k$ as fixed and omit it from the notation. The map $\nu: \mathbb{R}^{3} \rightarrow \mathbb{R}^{3}$ is given by

$$
\nu(x)=\left(x_{1} x_{2}, v\left(x_{1}\right), x_{3}\right)^{T},
$$

and the matrix $B$ is

$$
B=\left(\begin{array}{ccc}
-1 & 0 & 1 \\
-1 & 1 & 1 \\
1 & 0 & -1
\end{array}\right)
$$

with 1-dimensional kernel $\operatorname{ker} B=\operatorname{span}\left\{(1,0,1)^{T}\right\}$ and range $R=\operatorname{span}\left\{(0,1,0)^{T},(1,1,-1)^{T}\right\}$.

Assume that the rate $v(\cdot)$ is a smooth function with a unique zero $x_{1}^{*}>0$. The stationary states are therefore

$$
S_{+}=\left\{\left(x_{1}, x_{2}, x_{3}\right): x_{1} x_{2}=\lambda, \quad v\left(x_{1}\right)=0, \quad x_{3}=\lambda\right\}
$$

for $\lambda>0$, which is the part of the 1-dimensional affine space

$$
S=\left\{\left(x_{1}, x_{2}, x_{3}\right): x_{1}^{*} x_{2}-x_{3}=0, \quad x_{1}=x_{1}^{*}\right\}
$$

where all components are positive. The tangent space of $S$ at $x \in S$ is independent of $x$, namely

$$
T_{x} S=\operatorname{span}\left\{\left(0,1, x_{1}^{*}\right)^{T}\right\}
$$

We have

$$
D \nu(x)=\left(\begin{array}{ccc}
x_{2} & x_{1} & 0 \\
v^{\prime}\left(x_{1}\right) & 0 & 0 \\
0 & 0 & 1
\end{array}\right)
$$

and

$$
B D \nu(x)=\left(\begin{array}{ccc}
-x_{2} & -x_{1} & 1 \\
v^{\prime}\left(x_{1}\right)-x_{2} & -x_{1} & 1 \\
x_{2} & x_{1} & -1
\end{array}\right)
$$

Hence we see 
Proposition 6.1. The map $\tilde{B} \nu: \mathbb{R}^{3} \rightarrow R$ has 0 as a regular value if and only if $v^{\prime}\left(x_{1}^{*}\right) \neq 0$.

Now suppose that $v^{\prime}\left(x_{1}^{*}\right)=0$. This does not alter $S$ but does destroy the transversality of $\nu$ to ker $B$. If $x \in S_{+} \subset S$ then $T_{x} S$ is annihilated by $B D \nu(x)$ but not by $D \nu(x)$, so that $S_{+}$is normally degenerate with constant corank 1 . We choose a suitable complement $K_{x}$ to $T_{x} S$ in $\operatorname{ker} B D \nu(x)$. In the Appendix Section 7.2 it is shown that the reduced equation $\tilde{F}_{0}(u)=0$ (see Section 2) has uniform quadratic degeneracy provided $v^{\prime \prime}\left(x_{1}^{*}\right) \neq 0$. For studying bifurcations we are therefore in the context of Section 4.1 with $d=k=1$ and $m=2$.

\section{Appendix: calculations for examples in Section 6 .}

\subsection{Hamiltonian periodic orbits}

In this section we derive the condition (6.3).

Let $q(t)=r e(t) \in \mathbb{R}^{2}$ be a circular path with constant speed $|\dot{e}(t)|=\omega$. Let $u(t), v(t) \in \mathcal{H}$. Direct calculation shows that the Hessian of $\mathcal{J}$ at $q$ is

$$
\begin{aligned}
& D^{2} \mathcal{J}[q](u, v)=\int_{0}^{1} d t[\langle\dot{u}(t), \dot{v}(t)\rangle(E-V(r))]+ \\
& -\omega \int_{0}^{1} d t\left[\langle v(t), e(t)\rangle\left\langle\dot{u}(t), e^{\perp}(t)\right\rangle+\langle u(t), e(t)\rangle\left\langle\dot{v}(t), e^{\perp}(t)\right\rangle\right] r V^{\prime}(r)+ \\
& -\frac{\omega^{2}}{2} \int_{0}^{1} d t\left[r^{2}\left(V^{\prime \prime}(r)+\frac{1}{r} V^{\prime}(r)\right)\langle e(t), u(t)\rangle\langle e(t), v(t)\rangle+r V^{\prime}(r)\langle u(t), v(t)\rangle\right] .
\end{aligned}
$$

To compute $D^{2} \mathcal{J}$ at $q=q_{0}$ we set $r=r_{0}$ and use condition (6.2). We find

$$
\begin{aligned}
& D^{2} \mathcal{J}\left[q_{0}\right](u, v)=\int_{0}^{1} d t\left[\langle\dot{u}(t), \dot{v}(t)\rangle\left(E-V\left(r_{0}\right)\right)\right]+ \\
& -2 \omega \int_{0}^{1} d t\left[\left(\langle v(t), e(t)\rangle\left\langle\dot{u}(t), e^{\perp}(t)\right\rangle+\langle u(t), e(t)\rangle\left\langle\dot{v}(t), e^{\perp}(t)\right\rangle\right)\left(E-V\left(r_{0}\right)\right)\right]+ \\
& -\omega^{2} \int_{0}^{1} d t\left[\frac{1}{2}\left(r_{0}^{2} V^{\prime \prime}\left(r_{0}\right)+2\left(E-V\left(r_{0}\right)\right)\right)\langle e(t), u(t)\rangle\langle e(t), v(t)\rangle+\left(E-V\left(r_{0}\right)\right)\langle v(t), u(t)\rangle\right] .
\end{aligned}
$$

Since $E-V\left(r_{0}\right)>0$ the condition $D^{2} \mathcal{J}\left[q_{0}\right](u, v)=0$ is equivalent to

$$
\begin{gathered}
\int_{0}^{1} d t\left[\langle\dot{v}(t), \dot{u}(t)\rangle-2 \omega\left(\langle v(t), e(t)\rangle\left\langle\dot{u}(t), e^{\perp}(t)\right\rangle+\langle u(t), e(t)\rangle\left\langle\dot{v}(t), e^{\perp}(t)\right\rangle\right)+\right. \\
\left.-\left(\omega^{2} / 2\right) \Omega\langle e(t), u(t)\rangle\langle e(t), v(t)\rangle-\omega^{2}\langle v(t), u(t)\rangle\right]=0
\end{gathered}
$$

where

$$
\Omega:=\left(\frac{r_{0}^{2} V^{\prime \prime}\left(r_{0}\right)}{2\left(E-V\left(r_{0}\right)\right)}+1\right) .
$$


Integration by parts and a standard argument from calculus of variations shows that $u(t)$ lies in the kernel of $D^{2} \mathcal{J}\left[q_{0}\right]$ precisely when

$$
\begin{aligned}
\ddot{u}(t) & +2 \omega e(t)\left\langle\dot{u}(t), e^{\perp}(t)\right\rangle-2 \omega \frac{d}{d t}(\langle u(t), e(t)\rangle) e^{\perp}(t)+ \\
& +2 \omega^{2}\langle u(t), e(t)\rangle e(t)+\frac{\omega^{2}}{2} \Omega\langle e(t), u(t)\rangle e(t)+\omega^{2} u(t)=0 .
\end{aligned}
$$

Decomposing $u(t)$ as

$$
u(t)=a(t) e(t)+b(t) e^{\perp}(t)
$$

we find the equation (7.3) splits into

$$
\left\{\begin{array}{l}
\ddot{a}(t)+(4+\Omega / 2) \omega^{2} a(t)=0 \\
\ddot{b}(t)=0 .
\end{array}\right.
$$

Equations (7.4) imply that

$$
\operatorname{dim} \operatorname{ker} D^{2} \mathcal{J}\left[q_{0}\right]= \begin{cases}1 \text { if }(4+\Omega / 2) \neq n^{2} & \text { with } \quad n \in \mathbb{Z} \\ 2 \text { if }(4+\Omega / 2)=n^{2} & \text { for some } \quad n \in \mathbb{Z} .\end{cases}
$$

Finally, the expression (7.2) for $\Omega$ and condition (6.2) show that the condition $(4+\Omega / 2)=n^{2}$ is equivalent to (6.3).

\subsection{The reduced equation for the chemical reaction network}

Here we construct the reduced equation for (6.9) perturbed as in (6.8). From (6.9) we write (6.8) as

$$
F_{\varepsilon}(x):=B \nu(x)+\phi(\varepsilon, x)=f(x) w+v\left(x_{1}\right) e+\varepsilon \phi(\varepsilon, x)
$$

where $w=(1,1,-1)^{T}, e=(0,1,0)^{T}$ and $f(x)=-x_{1} x_{2}+x_{3} \in \mathbb{R}$. For $x \in S_{+}$the range $R_{x}$ of $B D \nu(x)$ is $\operatorname{span}\{w, e\}$ except if $v^{\prime}\left(x_{1}^{*}\right)=0$ in which case $R_{x}=\operatorname{span}\{w\}$. For $z \in \mathbb{R}_{+}^{3}$ we have

$$
\begin{aligned}
\left\langle w, F_{\varepsilon}(z)\right\rangle & =f(z)\|w\|^{2}+v\left(z_{1}\right)\langle w, e\rangle+\langle w, \phi(\varepsilon, z)\rangle \\
& =3 f(z)+v\left(z_{1}\right)+\langle w, \phi(\varepsilon, z)\rangle .
\end{aligned}
$$

For $x \in S_{+}$and $v^{\prime}\left(x_{1}^{*}\right)=0$ we have $\operatorname{ker} B D \nu(x)=u^{\perp}$ where $u=\left(x_{2}, x_{1},-1\right)^{T}$ and so it is natural for the Lyapunov-Schmidt reduction to take $L_{x}=\operatorname{span}\{u\}$. Accordingly for $z$ in a neighbourhood of $S_{+}$in $\mathbb{R}_{+}^{3}$ we write

$$
z=x+y n+\lambda u \in K_{x} \oplus L_{x}
$$

where $x \in S_{+}$and

$$
K_{x}=\left(T_{x} S \oplus \operatorname{span}\{u\}\right)^{\perp}=\operatorname{span}\{n\}
$$

where $n=\left(1+x_{1}^{* 2},-s x_{1}^{*}, s\right)^{T}$. By the IFT we can solve

$$
\left\langle w, F_{\varepsilon}(x+y n+\lambda u)\right\rangle=0
$$

in the form $\lambda=\lambda^{*}(x, y, \varepsilon)$ where $\lambda^{*}$ is a smooth function defined on a neighbourhood of $S_{0} \times 0$ in $\mathbb{R}^{3} \times \mathbb{R}^{q}$ and with $\frac{\partial \lambda^{*}}{\partial y}(x, 0,0)=0$ for all $x \in S$. It then remains to solve the reduced equation pair

$$
\left\langle s, F_{\varepsilon}\left(x+y n+\lambda^{*}(x, y, \varepsilon) u\right)\right\rangle=0, \quad\left\langle p, F_{\varepsilon}\left(x+y n+\lambda^{*}(x, y, \varepsilon) u\right)\right\rangle=0
$$


where $\operatorname{span}\{s\}=T_{x} S$ and $\operatorname{span}\{p\}=P_{x}$ chosen so that $P_{x}$ does not lie in $R_{x} \oplus T_{x} S$ (for example, $\left.p=(0,1,0)^{T}\right)$. Now

$$
\begin{aligned}
\left\langle s, F_{\varepsilon}(z)\right\rangle & =f(z)\langle s, w\rangle+v\left(z_{1}\right)\langle s, e\rangle+\varepsilon\langle s, \phi(\varepsilon, z)\rangle \\
& =a(s) v\left(z_{1}\right)+\varepsilon\langle b(s), \phi(\varepsilon, z)\rangle
\end{aligned}
$$

where

$$
a(s)=\langle s, e\rangle-\frac{1}{3}\langle s, w\rangle \quad \text { and } \quad b(s)=s-\frac{1}{3}\langle s, w\rangle w
$$

from (7.7), and similarly for $\left\langle p, F_{\varepsilon}(z)\right\rangle$. With $z=x+y n+\lambda^{*} u$ we can write

$$
\begin{aligned}
v\left(z_{1}\right)=v\left(x_{1}+y n_{1}+\lambda^{*} u_{1}\right) & =v\left(x_{1}^{*}+y\left(1+x_{1}^{* 2}\right)+\lambda^{*} x_{2}\right) \\
& =\frac{1}{2} v^{\prime \prime}\left(x_{1}^{*}\right)\left(y\left(1+x_{1}^{* 2}\right)+\lambda^{*} u_{1}\right)^{2}+O(3)
\end{aligned}
$$

where $O(3)$ terms are of third or higher order in $\left(y_{1}, \varepsilon\right)$ and smoothly parametrised by $x \in S_{+}$. Since $\lambda^{*}$ has no linear term in $y$ the smooth map $y_{1} \mapsto y\left(1+x_{1}^{* 2}\right)+\lambda^{*} u_{1}$ is a diffeomorphism germ at the origin in $K_{x}=\mathbb{R}$ smoothly parametrised by $x \in S_{+}$and sufficiently small $\varepsilon \in \mathbb{R}^{q}$. After we make this change of coordinate, the assumption $v^{\prime \prime}\left(x_{1}^{*}\right) \neq 0$ allows us to apply and a further local diffeomorphism in $K_{x}$ to absorb the $O(3)$ terms. We may therefore suppose without loss of generality that the equations (7.9) are

$$
\begin{aligned}
& a(s) c y_{1}^{2}+\varepsilon\langle b(s), \phi(\varepsilon, x, y)\rangle \\
& a(p) c y_{1}^{2}+\varepsilon\langle b(p), \phi(\varepsilon, x, y)\rangle
\end{aligned}
$$

where $c=\frac{1}{2} v^{\prime \prime}\left(x_{1}^{*}\right) \neq 0$, and so we have a quadratic system as claimed in Section 6.3. More specifically, the problem is of the form given in Example 2 in Section 5.3 and hence the branch points $x \in S_{+}$are given by the zeros (provided they are simple) of the function

$$
g(x):=a(s)\langle b(p), \phi(0, x, 0)\rangle-b(s)\langle a(p), \phi(0, x, 0)\rangle .
$$

\section{References}

1. R.Abraham and J.E.Marsden, Foundations of Mechanics (2nd ed), Benjamin/Cummings 1978.

2. A.Ambrosetti, Applications of critical point theory to variational problems on $R^{n}$, Proc. Int. Conf. Diff. Eqns.: Berlin 1999, v.1 (eds. B.Fiedler, K.Gröger, J.Sprekels), pp.469-484, World Scientific 2000.

3. A.Ambrosetti, V.Coti Zelati and I. Ekeland, Symmetry breaking in Hamiltonian systems, J. Diff. Eqns. 67 (1987), 165-184.

4. A.Ambrosetti and V.Coti Zelati, Perturbation of hamiltonian systems with Keplerian potentials, Math. Z. 201 (1989), 227-242.

5. V.I.Arnol'd, V.V.Goryunov, O.V.Lyashko and V.A.Vasil'ev. Singularity Theory I (=Encyclopaedia of Mathematical Sciences, Vol 6), Springer 1998 (Russian edition 1988).

6. V.A.Arnol'd, S.M.Gussein-Zade and A.N.Varchenko, Singularities of Differentiable Maps, Vol.1, Birkhäuser 1985.

7. T.Bartsch, Topological Methods for Variational Problems with Symmetries, Lecture Notes in Math. 1560, Springer-Verlag, Berlin 1993.

8. J.W.Bruce and P.J.Giblin, Curves and Singularities, CUP 1984.

9. K-C.Chang, Infinite Dimensional Morse Theory and Multiple Solution Problems, Birkhäuser 1993.

10. D.R.J.Chillingworth, Bifurcation from an orbit of symmetry, Singularities and Dynamical Systems (ed. S.N.Pnevmatikos), pp. 285-294, North-Holland 1985.

11. D.R.J.Chillingworth, Bifurcation from a manifold, Singularity Theory and its Applications (Warwick 1989, Part II), (eds. M.Roberts and I.Stewart), pp. 22-37, Lecture Notes in Math. 1463, Springer-Verlag, Berlin 1991.

12. D.R.J.Chillingworth, The Signorini perturbation scheme in an abstract setting, Proc. Roy. Soc. Edinburgh, 119A (1991), 373-395. 
13. D.R.J.Chillingworth, Generic multiparameter bifurcation from a manifold, Dyn. Stab. Systems, 15 (2000), 101137.

14. C.Chicone, Bifurcations of nonlinear oscillations and frequency entrainment near resonance, SIAM J. Math. Anal. 23 (1992), 1577-1608.

15. C.Chicone, Periodic solutions of a system of coupled oscillators near resonance, SIAM J. Math. Anal. 26 (1995), 1257-1283.

16. C.Chicone, A geometric approach to regular perturbation theory with an application to hydrodynamics, Trans. Amer. Math. Soc. 347 (1995), 4559-4598.

17. D.P.L.Castrigiano and S.A.Hayes, Catastrophe Theory, Addison-Wesley, Reading, Mass. 1993.

18. S.-N.Chow and J.K Hale, Methods of Bifurcation Theory, Springer 1982.

19. E.N.Dancer, On the existence of bifurcating solutions in the presence of symmetries, Proc. Roy. Soc. Edinburgh 85A (1980), 321-336.

20. E.N.Dancer, The $G$-invariant implicit function theorem in infinite dimensions, Proc. Roy. Soc. Edinburgh 92A (1982), 13-30.

21. E.N.Dancer, Perturbation of zeros in the presence of symmetries, J. Austral. Math. Soc. (Ser. A) 36 (1984), 106-125.

22. M.Demazure, Bifurcations and Catastrophes, Springer 2000.

23. M.Domijan and M.Kirkilionis, Graph theory and qualitative analysis of reaction networks, Networks and Heterogenous Media 3 (2008), 295-322.

24. J.P.Dufour, Familles de courbes planes différentiables, Topology 22 (1983), 449-474.

25. M.J.Field, Symmetry Breaking for Compact Lie Groups, Memoirs of the AMS 574, 1996.

26. M.J.Field, Lectures on bifurcations, dynamics and symmetry, Research Notes in Math. 356, Pitman/Longman 1996.

27. B.Fiedler, S.Liebscher and J.C.Alexander, Generic Hopf bifurcation from lines of equilibria without parameters, I Theory, J.Diff.Eqns. 167 (2000), 16-35.

28. C.G.Gibson, Singular Points of Smooth Mappings, Research Notes in Math. 25, Pitman 1979.

29. M.Golubitsky and V.Guillemin, Stable Mappings and their Singularities, Springer-Verlag, New York 1973.

30. M.Golubitsky, D.G.Schaeffer and I.Stewart, Singularities and Groups in Bifurcation Theory II, Springer-Verlag, New York 1988.

31. J.K.Hale, Generic bifurcation with applications, in Nonlinear analysis and mechanics: Heriot-Watt Symposium Vol.I (ed. R.J.Knops), pp. 59-157, Research Notes in Math. 17, Pitman, London 1977.

32. J.K.Hale and P.Z.Taboas, Bifurcation near degenerate families, Applicable Anal. 11 (1980), 21-37.

33. M.Krupa, Bifurcations of relative equilibria, SIAM J. Math. Anal. 21 (1990), 1453-1486.

34. R.J.Magnus, On perturbations of a translationally invariant differential equation, Proc. Roy. Soc. Edinburgh 110A (1988), 1-25.

35. D.Mond, On the classification of germs of maps from $\mathbb{R}^{2}$ to $\mathbb{R}^{3}$, Proc.London Math.Soc. 50 (1985), 333-369.

36. J.Moser, Periodic orbits near an equilibrium and a theorem by Alan Weinstein, Comm. Pure Appl. Math. 29 (1976), 727-747.

37. T.Poston and I.Stewart, Catastrophe Theory and its Applications, Pitman, London 1978.

38. M.B.H.Rhouma and C.Chicone, On the continuation of periodic orbits, Methods Appl. Anal. 7 (2000), 85-104.

39. M.Reeken, Stability of critical values and isolated critical continua, Manuscripta Math. 12 (1974), 163-193.

40. J.H.Rieger, Families of maps from the plane to the plane, J. London Math. Soc. 36 (1987), 351-369.

41. L.Sbano, Periodic Orbits of Hamiltonian Systems Encyclopedia of Complexity and Systems Science Entry n.73, 2009

42. A.Vanderbauwhede, Symmetry and bifurcations near families of solutions, J. Diff. Eqns. 36 (1980), 173-187.

43. A.Weinstein, Perturbation of periodic manifolds of Hamiltonian systems, Bull. Amer. Math. Soc. 77 (1971), 814-818. 
D.R.J. Chillingworth

School of Mathematics,

University of Southampton,

Southampton SO17 1BJ, UK

d.r.chillingworth@soton.ac.uk

L. Sbano

Mathematics Institute,

University of Warwick,

Coventry CV4 7AL, UK

sbano@maths.warwick.ac.uk

luca.sbano@istruzione.it 